\title{
Crosstalk Between Autophagy and Ferroptosis and Its Putative Role in Ischemic Stroke
}

\author{
Jie Liu'1,2,3t, Zhen-Ni Guo ${ }^{1,2+}$, Xiu-Li Yan', Shuo Huang ${ }^{1,2,3}$, Jia-Xin Ren ${ }^{1,2}$, Yun Luo ${ }^{1,2,3}$ and \\ Yi Yang ${ }^{1,2,3 *}$ \\ ' Department of Neurology, Stroke Center \& Clinical Trial and Research Center for Stroke, The First Hospital of Jilin University, \\ Changchun, China, ${ }^{2}$ China National Comprehensive Stroke Center, Changchun, China, ${ }^{3}$ Jilin Provincial Key Laboratory of \\ Cerebrovascular Disease, Changchun, China
}

\section{OPEN ACCESS}

Edited by:

Marco Bacigaluppi,

San Raffaele Scientific Institute

(IRCCS), Italy

Reviewed by:

Ishraq Alim,

Cornell University, United States

Sheng Zhang,

University of Texas Health Science Center at Houston, United States

*Correspondence:

Yi Yang

doctoryangyi@163.com

${ }^{\dagger}$ These authors have contributed equally to this work

Specialty section:

This article was submitted to Cellular Neuropathology, a section of the journal

Frontiers in Cellular Neuroscience

Received: 29 June 2020

Accepted: 14 September 2020

Published: 02 October 2020

Citation:

Liu J, Guo Z-N, Yan X-L, Huang S, Ren J-X, Luo Y and Yang $Y$ (2020) Crosstalk Between Autophagy and Ferroptosis and Its Putative Role

in Ischemic Stroke.

Front. Cell. Neurosci. 14:577403.

doi: 10.3389/fncel.2020.577403
Autophagy is a conserved process to maintains homeostasis via the degradation of toxic cell contents, which can either promote cell survival or accelerate cellular demise. Ferroptosis is a recently discovered iron-dependent cell death pathway associated with the accumulation of lethal reactive lipid species. In the past few years, an increasing number of studies have suggested the crosstalk between autophagy and ferroptosis. Ischemic stroke is a complex brain disease regulated by several cell death pathways, including autophagy and ferroptosis. However, the potential links between autophagy and ferroptosis in ischemic stroke have not yet been explored. In this review, we briefly overview the mechanisms of ferroptosis and autophagy, as well as their possible connections in ischemic stroke. The elucidation of crosstalk between different cell death pathways may provide insight into new future ischemic stroke therapies.

Keywords: autophagy, ferroptosis, cell death, ischemic stroke, iron overload, lipid peroxidation, reactive oxygen species

\section{INTRODUCTION}

Stroke is one of the major causes of death and disability worldwide (Haley et al., 2019), and includes two main subtypes: ischemic stroke and hemorrhagic stroke. Ischemic stroke results from a lack of blood supply to the brain, and accounts for approximately $85 \%$ of all cases of stroke. Nowadays, several cell death pathways have been identified to be involved in ischemic stroke pathophysiology, including apoptosis, necrosis, and autophagy. Among these, necrosis has been generally regarded as a passive and uncontrolled form of cell death, while more recently, certain types of regulated necrosis have also been found, such as necroptosis, pyroptosis, ferroptosis, parthanatos, and CypDmediated necrosis. Interventions targeting these specific types of regulatory necrosis have provided new ideas for the treatment of ischemic stroke (Lu et al., 2020).

Autophagy is an evolutionarily conserved process to degrade toxic proteins, damaged organelles, and invading pathogens via the lysosomal pathway. At the molecular level, autophagy is mainly executed by multiple autophagy-related genes (Atg), and is also regulated by a complex signaling network. Autophagy plays an important role in maintaining cellular homeostasis, regulating organism growth, and modulating the development of diseases (Doria et al., 2013). In ischemic stroke, it can either postpone or accelerate cell death, depending on the degree of activation.

Ferroptosis is iron-dependent regulated necrosis associated with excess reactive lipid species, due to accumulated lipid peroxidation. Recently, studies found that ferroptosis also plays an 
important role in the development of ischemic stroke through influencing iron metabolism or lipid peroxidation, whereas inhibiting ferroptosis successfully reverses ischemic damages (Tuo et al., 2017; Alim et al., 2019; Guan et al., 2019). Furthermore, ferroptosis is significantly distinct from other forms of cell death, including autophagy, in terms of cell morphological characteristics, biological features and so on (Dixon et al., 2012; Klionsky et al., 2016; Xie et al., 2016; Zille et al., 2017). Conventionally, autophagy-dependent cell death is characterized by the formation of autophagosomes, which fuse with lysosomes to form autolysosomes. Differently, ferroptosis is featured by an intact cell membrane and normal nucleus, but shrinking mitochondria with increased membrane densities, reduced even disappeared mitochondria crista and ruptured outer membrane. For detailed information regarding the difference between autophagy and ferroptosis (see Table 1).

While autophagy and ferroptosis are mechanistically and morphologically distinct cell death pathways, an increasing number of studies have recently reported significant crosstalk between them (Kang and Tang, 2017; Zhou et al., 2019; Liu et al.,

TABLE 1 | Characteristics of autophagy and ferroptosis.

\begin{tabular}{|c|c|c|}
\hline & Autophagy & Ferroptosis \\
\hline $\begin{array}{l}\text { Morphological } \\
\text { characteristics }\end{array}$ & $\begin{array}{l}\text { Formation of } \\
\text { autophagosomes, a double } \\
\text { membrane vesicle } \\
\text { containing multiple } \\
\text { cytoplasmic contents. The } \\
\text { formed autophagosomes } \\
\text { fuse with lysosomes to } \\
\text { form autolysosomes }\end{array}$ & $\begin{array}{l}\text { Atrophy of mitochondria } \\
\text { with increased membrane } \\
\text { densities, reduced even } \\
\text { disappeared mitochondria } \\
\text { crista and ruptured outer } \\
\text { membrane, normal nucleus }\end{array}$ \\
\hline $\begin{array}{l}\text { Development } \\
\text { steps }\end{array}$ & $\begin{array}{l}\text { Iron overload, GSH } \\
\text { depletion and Gpx4 } \\
\text { inactivation, lipid } \\
\text { peroxidation, and impaired } \\
\text { system Xc- }\end{array}$ & $\begin{array}{l}\text { Initiation and nucleation of } \\
\text { autophagosomes, } \\
\text { maturation of } \\
\text { autophagosomes and } \\
\text { fusion of autophagosomes } \\
\text { with the lysosome and } \\
\text { degradation }\end{array}$ \\
\hline Key regulators & $\begin{array}{l}\text { Atgs, mTOR, AMPK, } \\
\text { BECN1, PI3K, p62, p53, } \\
\text { ULK1, TFEB }\end{array}$ & $\begin{array}{l}\text { Positive regulator: SAT1, } \\
\text { GLS2, p53, ACSL4, TfR1 } \\
\text { Negative regulator: GSH, } \\
\text { NRF2, HSPA5, NCOA4, } \\
\text { HSPB1, Gpx4, SLC7A11 }\end{array}$ \\
\hline $\begin{array}{l}\text { Common } \\
\text { inducers and } \\
\text { inhibitors }\end{array}$ & $\begin{array}{l}\text { Inducer: Rapamycin, } \\
\text { Brefeldin A, Tunicamycin, } \\
\text { starvation media } \\
\text { Inhibitor: 3-MA, Bafilomycin } \\
\text { A1 }\end{array}$ & $\begin{array}{l}\text { Inducer: Erastin, RSL3, } \\
\text { Sorafenib, SAS, FIN56, } \\
\text { FAC } \\
\text { Inhibitor: DFO, Fer-1, } \\
\text { Vitamin E, Liproxststatin-1, } \\
\text { 2,2-BP, DFA, ciclopirox } \\
\text { olamine }\end{array}$ \\
\hline
\end{tabular}

Atgs, autophagy-related genes; AMPK, Adenosine 5'-monophosphate (AMP)activated protein kinase; BECN1, beclin 1; PI3K, Phosphatidylinositol 3-kinase; TFEB, Transcription factor EB; 3-MA, 3-Methyladenine; GLS2, Glutaminase 2; ACSL4, Acyl-COA synthetase long chain family member 4; TfR1, Transferrin receptor 1; GSH, glutathione; NRF2, nuclear factor E2 related factor 2; HSPA5, Heat shock 70kDa protein 5; NCOA4, Nuclear receptor coactivator 4; HSPB1, heat shock protein family $B$ (small) member 1; Gpx4, glutathione peroxidase 4; SLC7A11, solute carrier family 7 member 11; RSL3, Ras-selective lethal small molecule 3; SAS, sulfasalazine; FAC, ferric ammonium citrate; DFO, deferoxamine; Fer-1, Ferrostatin-1.
2020). This identification not only favors a deeper understanding of cell death, but also provides new ideas for the regulation of disease and development of therapeutic strategies. In this review, we briefly introduce the mechanisms of autophagy and ferroptosis, as well as pathways that mediate their interactions. On this basis, we further discuss their possible interrelationships in ischemic stroke.

\section{AUTOPHAGY AND ISCHEMIC STROKE}

\section{Mechanisms of Autophagy}

Autophagy can be divided into three general subtypes: macroautophagy, microautophagy, and chaperone-mediated autophagy (Klionsky et al., 2016). Macroautophagy is a continuous and dynamic process initiated by the formation of autophagosomes, which are double membrane vesicles that contain multiple cytoplasmic components, including damaged organelles and dysfunctional proteins. The formed autophagosomes then fuse with lysosomes to form autolysosomes, and induce the degradation and recycling of cellular components. This process allows cells to maintain homeostasis under stressful conditions.

So far, more than 30 Atg proteins have been found to participate in the execution of autophagy. The formation of autophagosomes is considered to be regulated by two macromolecular complexes, the ULK1 complex (ULK1/Atg1mTOR-Atg13-RB1CC1/FIP200), which is responsible for the initiation of autophagy, and the PtdIns3K complex (PIK3C3/VPS34- Beclin 1-Atg14), which is responsible for the nucleation of autophagy (Xie et al., 2015). ULK1 kinase can also recruit PtdIns $3 \mathrm{~K}$ complex by phosphorylating some of the components, which results in the production of phosphatidylinositol 3-phosphate [PI(3)P] and favors autophagosomal membrane nucleation (Russell et al., 2013). In addition, Atg5 and Atg12 have been showed to cooperate with Atg7, forming the Atg5-Atg12-Atg16-like 1 (Atg16L1) complex, which facilitates the elongation and expansion of autophagosome membranes to form a completely closed autophagosome (Nakatogawa, 2013), while the formation of microtubule-associated protein 1 light chain 3 (LC3)phosphatidyl ethanolamine (PE) conjugate is also required in this process. Fujita et al. proposed that the Atg16L1 complex may function as a scaffold for LC3 lipidation and affect the sites of autophagosome synthesis (Fujita et al., 2008). Finally, the fusion of autophagosomes with lysosomes is also wellregulated, wherein hairpin-type tail-anchored SNARE syntaxin 17, pleckstrin homology domain containing protein family member 1 (PLEKHM1), and Atg14 have been identified as important regulators.

In addition, several complicated signaling pathways could also play important roles in autophagy regulation (Behrends et al., 2010; Mizushima and Komatsu, 2011). For example, the mTOR complex 1 (mTORC1) and AMP-activated protein kinase (AMPK), are well-known upstream regulators of autophagy. In nutrient-rich conditions, mTORC1 is overactivated, which then suppresses autophagy by directly binding and phosphorylating 
ULK1 (Hosokawa et al., 2009). While in nutrient-depleted conditions, AMPK is upregulated, which promotes the activation of ULK1 kinase complex by inactivating mTORC1 or dephosphorylating ULK1 (Egan et al., 2011), the activated ULK1 then induces Atg13 phosphorylation and autophagy.

BECN1 (beclin 1) is well-known as a key autophagy modulator; its effects depend on its binding proteins. For example, BECN1, which binds to core components of the Class III PI3K complex, promotes the formation of autophagosomes (Liang et al., 1999; Kihara et al., 2001). The BH3 structure of the BECN1, which binds to the antiapoptotic protein $\mathrm{Bcl}-2 / \mathrm{Bcl}-$ $\mathrm{xl}$, inhibits the occurrence of autophagy, while downregulating Bcl-2 activates the autophagic pathway (Lian et al., 2011; Yang and Yao, 2015).

Phosphatidylinositol 3-kinase (PI3K) is another important regulator involved in phagosome maturation and autophagy (Thi and Reiner, 2012), which can be divided into three classes. Class I PI3K has been shown to inhibit autophagy through the PI3KAkt-TSCl/TSC2-mTOR pathway (Hawkins et al., 2006; Pilli et al., 2012), and S14161, a Class I PI3K inhibitor, induces autophagy by regulating the Beclin-1/Vps34 complex (Wang et al., 2017). Besides, apelin-13, an adipokine, inhibit foam cell formation by activating autophagy via the Class III PI3K/Beclin-1 pathway (Yao et al., 2015).

The ubiquitin-binding protein, p62, also known as sequestosome1 (SQSTM1), is also involved in autophagy. Conventionally, p62 is considered as a cargo receptor recruiting/sequestering the ubiquitinated cargo to target autophagosomes and then degrade within lysosomes. This process can be suppressed by the Class III PI3K inhibitors, or consumption of the Atg12 protein homolog (Kim et al., 2008). Recently, it has also been found to play a more complicated role via regulating various signaling pathways including the mTORC1 pathway (Moscat et al., 2016); p62 deficiency impairs the translocation of mTORC1 to the lysosomes and its activation in response to amino acids and Tsc1 ablation (Duran et al., 2011; Umemura et al., 2016).

\section{The Dual Effects of Autophagy in Ischemic Stroke}

The induction of autophagy has been identified in neurons, glia cells, brain microvascular cells, and other cell types after ischemic stroke (Wang P. et al., 2018). It is generally believed that autophagy can play a dual role in ischemic stroke (Table 2); moderate activation of autophagy could enable neuronal cell survival, while excessive autophagy triggers neuronal death. For example, mitophagy can induce mitochondrial clearance and the inhibition of apoptosis, which represents the neuroprotective effect of autophagy (Zhang et al., 2014; Shen et al., 2017). Sirtuin3 (Sirt3), upregulated by oxygen and glucose deprivation, increases autophagy through regulating the AMPK-mTOR pathway, and then plays a protective role in neuronal ischemia (Dai et al., 2017). Metastasis-associated lung adenocarcinoma transcript 1 (MALAT1) long non-coding RNA (lncRNA) also activates autophagy and protects against cerebral ischemia by binding to miR-200c-3p and upregulating
Sirt1 expression (Wang et al., 2019). On the other hand, excess autophagy also contributes to endothelial damage and destruction of the blood-brain barrier (BBB) under ischemic conditions. Activation of the autophagy-lysosomal pathway after ischemia promotes degradation of the $\mathrm{BBB}$ component claudin-5, while the inhibition of autophagy prevents damage to brain microvascular endothelial cells during reperfusion (Yang G. et al., 2018). Similarly, the absence of the circadian clock protein period1 (PER1) suppresses hippocampal autophagy and leads to vulnerability during ischemic stroke (Rami et al., 2017). So far, modulations of autophagy intensity have been reported as feasible strategies in the treatment of ischemic stroke. Drugs such as dexmedetomidine have been found to protect neurons from ischemic damage by promoting autophagy (Luo et al., 2017), and some non-coding RNAs targeting autophagy have also been shown to play important roles in ischemic stroke (Wang N. et al., 2018; Yu et al., 2019).

\section{FERROPTOSIS AND ISCHEMIC STROKE}

Ferroptosis is a recently discovered regulated form of cell death based on iron-dependent lipid peroxidation. In general, the induction of ferroptosis can be divided into four critical events: (1) iron overload, (2) glutathione (GSH) depletion and glutathione peroxidase $4(\mathrm{Gpx} 4)$ inactivation, (3) lipid peroxidation, and (4) impaired system Xc-. These events form positive feedback loops and generally push cells toward death. Stopping any critical events would stop the co-dependent events and then suppress the occurrence of ferroptosis. We now briefly introduce the relationship between these events and their potential roles in ischemic stroke (Figure 1 and Table 2).

\section{Iron Overload}

It is well-known that iron metabolism plays an important role in the brain. In general, iron-loaded transferrin (holotransferrin) transports iron to the brain through the endothelial cells of the $\mathrm{BBB}$ and the choroid plexus epithelium. Physiologically, the brain can be sheltered from fluctuations in systemic iron due to the protection provided by the BBB. However, under acute ischemic conditions, the BBB is disrupted, which allows the entry of free iron and ferritin into the brain parenchyma, converting hydrogen peroxide to hydroxyl radicals via the Fenton reaction (Kell, 2009; Chen et al., 2011). This process significantly increases the generation of reactive oxygen species (ROS), which promotes nucleic, proteomic, and membrane damage, and finally mediates ferroptotic cell death (DeGregorio-Rocasolano et al., 2019). Nowadays, iron overload has been identified as a major source of oxidative stress in ischemic brains (Carbonell and Rama, 2007). Furthermore, in the early stage of reperfusion, it also increases the risk of hemorrhagic transformation, and thereby exaggerates the poor outcomes associated with cerebral ischemia (García-Yébenes et al., 2018). In clinical studies, high levels of serum ferritin also offset the beneficial effect of thrombolytic therapies in ischemic stroke patients (Millan et al., 2007). These studies 
TABLE 2 | Autophagy and ferroptosis in ischemic stroke.

\begin{tabular}{|c|c|c|c|c|}
\hline References & Interventions & Subjects & Targets & Effects \\
\hline \multicolumn{5}{|l|}{ Harmful autophagy } \\
\hline Feng et al. (2017) & Melatonin & MCAO mice & ER stress $\downarrow$ & $\begin{array}{l}\text { Melatonin protects against cerebral ischemia through inhibiting ER } \\
\text { stress-dependent autophagy. }\end{array}$ \\
\hline Bu et al. (2014) & W007B & MCAO rats & $\begin{array}{l}\text { Beclin-1, LC3B-II } \downarrow \\
\text { p62 } \uparrow\end{array}$ & W007B protects against cerebral ischemia through inhibiting autophagy. \\
\hline Baek et al. (2014) & Carnosine & MCAO rats & $\begin{array}{l}\text { LC3-II formation } \downarrow \\
\text { P-p70S6K, p-mTOR } \uparrow\end{array}$ & $\begin{array}{l}\text { Carnosine protects against cerebral ischemia at least partially by } \\
\text { attenuating deleterious autophagy. }\end{array}$ \\
\hline Jiang et al. (2017) & $\mathrm{NaHS}$ & MCAO rats & $\begin{array}{l}\text { LC3 } 11 / I \downarrow \\
\text { P62 } \uparrow \\
\text { Autophagolysosomes } \downarrow\end{array}$ & $\begin{array}{l}\text { NaHS protects against cerebral ischemia by inhibiting overactivated } \\
\text { autophagy. }\end{array}$ \\
\hline Li et al. (2012) & TMEM166 siRNA & MCAO rats & Beclin-1, LC3 $\downarrow$ & $\begin{array}{l}\text { TMEM166 siRNA protects against cerebral ischemia by inhibiting } \\
\text { TMEM166-induced autophagy. }\end{array}$ \\
\hline Luo et al. (2017) & DEX & $\begin{array}{l}\text { MCAO mice, OGD primary } \\
\text { cultured neurons }\end{array}$ & $\begin{array}{l}\text { Bcl-1, p62, HIF-1 } \alpha \uparrow \\
\text { LC3, Beclin-1 } \downarrow\end{array}$ & $\begin{array}{l}\text { DEX protects against cerebral ischemia via inhibition of neuronal } \\
\text { autophagy by upregulation of HIF-1 } \alpha \text {. }\end{array}$ \\
\hline Yang G. et al. (2018) & HSYA & OGD primary BMECs & $\begin{array}{l}\text { Autophagosomes } \downarrow \\
\text { LC3, Beclin- } 1 \downarrow \\
\text { P-Akt, p-mTOR } \uparrow\end{array}$ & $\begin{array}{l}\text { HSYA protects against OGD by inhibiting autophagy via the Class I } \\
\text { PIJK/Akt/mTOR signaling pathway. }\end{array}$ \\
\hline \multicolumn{5}{|c|}{ Protective autophagy } \\
\hline Qi et al. (2015) & $\mathrm{RIC}$ & MCAO rats & $\begin{array}{l}\text { P-Bcl-2 } \uparrow \\
\text { Bcl-2/Beclin1 complex } \downarrow\end{array}$ & $\begin{array}{l}\mathrm{RIC} \text { triggers autophagy and reduces mitochondrial damage after } \\
\text { cerebral ischemia }\end{array}$ \\
\hline Li et al. (2014) & Rapamycin & MCAO rats & $\begin{array}{l}\text { LC3-II and Beclin- } 1 \text { in the } \\
\text { mitochondria } \uparrow \\
\text { P62 translocation to the } \\
\text { mitochondria } \uparrow\end{array}$ & $\begin{array}{l}\text { Rapamycin attenuates mitochondrial dysfunction following cerebral } \\
\text { ischemia, which is linked to enhanced mitophagy. }\end{array}$ \\
\hline Zhang et al. (2014) & TM and TG & MCAO mice & ER stress $\uparrow$ & $\begin{array}{l}\text { TM and TG protects against cerebral ischemia via inducing ER stress, } \\
\text { which are based on the PARK2-mediated mitophagy. }\end{array}$ \\
\hline Wang et al. (2014b) & ARRB1 & OGD neurons & Autophagosome $\uparrow$ & $\begin{array}{l}\text { ARRB1 protects against OGD through coordination of } \\
\text { BECN1-dependent autophagy. }\end{array}$ \\
\hline Dai et al. (2017) & / & OGD neurons & $\begin{array}{l}\text { Beclin-1, LC3-II } \uparrow \\
\text { P-AMPK } \uparrow \\
\text { P-mTOR } \downarrow\end{array}$ & $\begin{array}{l}\text { Sirt3 protects against OGD by inducing autophagy through regulation of } \\
\text { the AMPK-mTOR pathway. }\end{array}$ \\
\hline Gao et al. (2015) & IPC & OGD neurons & P-Akt, LC3-II/LC3-I & $\begin{array}{l}\text { IPC may attenuate ischemic injury in neurons through induction of } \\
\text { Akt-independent autophagy. }\end{array}$ \\
\hline Shen et al. (2017) & APC & $\begin{array}{l}\text { MCAO mice } \\
\text { OGD neurons }\end{array}$ & $\begin{array}{l}\text { TOMM20, COX4l1 } \downarrow \\
\text { PARK2 translocation to the } \\
\text { mitochondria } \uparrow\end{array}$ & $\begin{array}{l}\text { PARK2-induced mitophagy is required for the APC-mediated } \\
\text { neuroprotection to ischemic injury, which also extends the reperfusion } \\
\text { window of cerebral ischemia. }\end{array}$ \\
\hline Wang et al. (2019) & MALAT1 IncRNAs & OGD BMECs & $\begin{array}{l}\text { MALAT1, Sirt1 } \uparrow \\
\text { MiR-200c-3p } \downarrow\end{array}$ & $\begin{array}{l}\text { MALAT1 IncRNAs activates autophagy and protects against OGD by } \\
\text { binding to miR-200c-3p and upregulating Sirt1 expression. }\end{array}$ \\
\hline
\end{tabular}




\begin{tabular}{|c|c|c|c|c|}
\hline References & Interventions & Subjects & Targets & Effects \\
\hline \multicolumn{5}{|l|}{ Ferroptosis } \\
\hline Ahmad et al. (2014) & SES & MCAO mice & $\begin{array}{l}\text { GSH } \uparrow \\
\text { MAPK/ERK, P38 } \downarrow \\
\text { Superoxide radical } \downarrow \\
\text { Lipid peroxidation } \downarrow\end{array}$ & $\begin{array}{l}\text { SES induces neuroprotection by ameliorating lipid peroxidation and increased } \\
\text { GSH activity following cerebral ischemia. }\end{array}$ \\
\hline Guan et al. (2019) & Carvacrol & $\begin{array}{l}\text { OGD neurons } \\
\text { Gerbils with bilateral carotid } \\
\text { artery ligation }\end{array}$ & $\begin{array}{l}\text { Lipid peroxide } \downarrow \\
\text { Gpx4 } \uparrow\end{array}$ & $\begin{array}{l}\text { Carvacrol protects against ischemic stroke by inhibiting ferroptosis through } \\
\text { increasing the expression of Gpx4. }\end{array}$ \\
\hline Alim et al. (2019) & Selenium & MCAO mice & $\begin{array}{l}\text { TFAP2c, Sp1 } \uparrow \\
\text { Gpx4 } \uparrow\end{array}$ & $\begin{array}{l}\text { Pharmacological Se supplementation protects cells from ferroptosis in ischemic } \\
\text { stroke via increasing GPX4 expression. }\end{array}$ \\
\hline Liu et al. (2017) & DMED & $\begin{array}{l}\text { OGD PC12 and primary } \\
\text { neuronal cells }\end{array}$ & SOD, GSH-Px $\downarrow$ & DMED protects against OGD depending on its anti-oxidative activity. \\
\hline García-Yébenes et al. (2012) & Iron-fed diet & MCAO mice & Iron accumulation $\uparrow$ & $\begin{array}{l}\text { Iron-fed diet increases ischemic damage and HT by increasing brain iron } \\
\text { accumulation. }\end{array}$ \\
\hline Hanson et al. (2009) & DFO & MCAO rats & Iron accumulation $\downarrow$ & Intranasal DFO treatment decreases infarct volume by inhibiting iron overload. \\
\hline Tuo et al. (2017) & / & $\begin{array}{l}\mathrm{Tau}^{-/-} \text {young and aged } \\
\text { MCAO mice and rats }\end{array}$ & Intensity of iron accumulation & $\begin{array}{l}\text { Tau suppression induced by cerebral ischemic prevent ferroptosis in young } \\
\text { tau-/- mice, while the protective benefit of tau-/- was negated in older mice due } \\
\text { to the accelerated age-dependent brain iron accumulation. }\end{array}$ \\
\hline García-Yébenes et al. (2018) & Iron-fed diet & $\begin{array}{l}\text { Mice subjected to } \\
\text { thromboembolic stroke } \\
\text { treated with tPA }\end{array}$ & $\begin{array}{l}\text { Lipid peroxidation } \uparrow \\
\text { Iron accumulation } \uparrow\end{array}$ & $\begin{array}{l}\text { Iron-fed mice show less neuroprotection after tPA administration. Iron overload } \\
\text { also exacerbates the risk of HT after early tPA administration enhanced basal } \\
\text { serum lipid peroxidation. }\end{array}$ \\
\hline Lan et al. (2020) & NTE & MCAO rats & $\begin{array}{l}\text { TFR1, DMT1 } \downarrow \\
\text { Iron accumulation } \downarrow \\
\text { SLC7A11, Gpx4, GSH } \uparrow\end{array}$ & $\begin{array}{l}\text { NTE treats ischemic injury by inhibiting ferroptosis through the TFR1/DMT1 and } \\
\text { SCL7A11/Gpx4 pathways. }\end{array}$ \\
\hline DeGregorio-Rocasolano et al. (2018) & ATf & $\begin{array}{l}\text { MCAO rats } \\
\text { OGD primary neuronal cells }\end{array}$ & $\begin{array}{l}\text { TSAT, HTf uptake } \downarrow \\
4-\mathrm{HNE} \downarrow\end{array}$ & $\begin{array}{l}\text { ATf reduces neuronal damage by preventing NMDA-induced HTf uptake and } \\
\text { ROS production. }\end{array}$ \\
\hline Papazisis et al. (2008) & DFO & $\begin{array}{l}\text { Neonatal rats with } \\
\text { hypoxia-ischemia }\end{array}$ & $\begin{array}{l}\text { Iron accumulation } \downarrow \\
\text { Glutamate, aspartate } \downarrow\end{array}$ & $\begin{array}{l}\text { DFO decreases the excitatory amino acid levels and improves the histological } \\
\text { outcome after hypoxia-ischemia. }\end{array}$ \\
\hline Millan et al. (2007) & / & $\begin{array}{l}\text { Patients with acute } \\
\text { ischemic stroke treated } \\
\text { with tPA }\end{array}$ & Intensity of iron accumulation & $\begin{array}{l}\text { Increased body iron stores are associated with poor outcome and symptomatic } \\
\text { HT. Iron overload may offset the beneficial effect of thrombolytic therapies. }\end{array}$ \\
\hline Pekcec et al. (2013) & / & MCAO mice & Sema3A, 12/15-LOX $\uparrow$ & Sema3A increases cortical damage, which is reversed by $12 / 15$-LOX inhibition. \\
\hline van Leyen et al. (2006) & Baicalein & MCAO mice & $12 / 15-$ LOX $\downarrow$ & $\begin{array}{l}\text { Baicalein protects against cerebral ischemia by inhibiting the 12/15-LOX } \\
\text { pathway. }\end{array}$ \\
\hline Yigitkanli et al. (2013) & LOXBlock-1 & MCAO mice & $12 / 15-$ LOX $\downarrow$ & LOXBlock-1 protects against ischemic stroke by inhibiting lipid peroxidation. \\
\hline
\end{tabular}




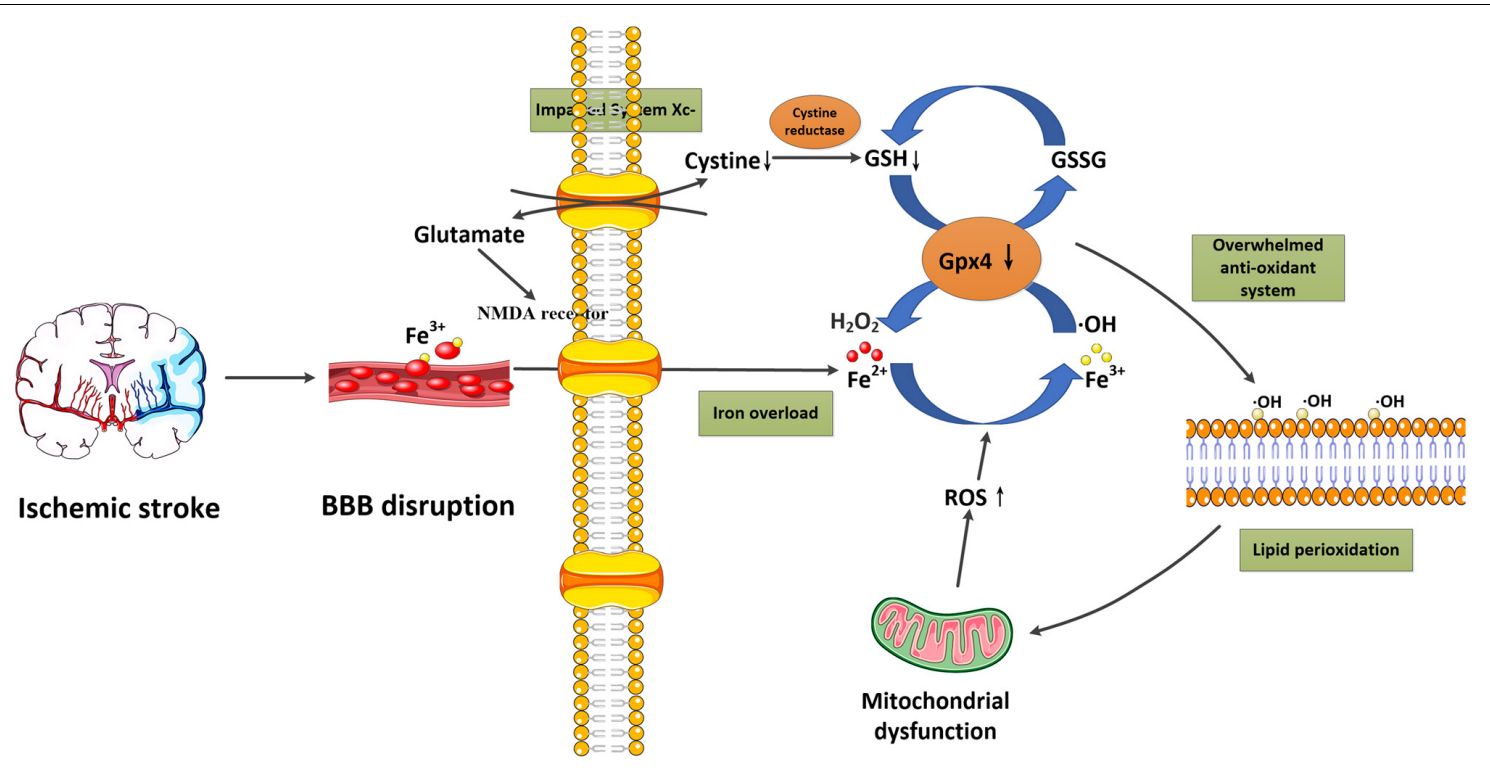

FIGURE 1 | Schematic overview of ferroptosis in ischemic stroke. Following ischemic stroke, the BBB is disrupted, which allows a large amount of ferritin-containing $\mathrm{Fe}^{3+}$ in the blood to be released into the brain parenchyma. This converts hydrogen peroxide to hydroxyl radicals via the Fenton reaction. Meanwhile, system Xc- is simultaneously impaired, which inhibits cystine-glutamate exchange and decreases the generation of the antioxidant GSH, and reduces its oxidation to GSSG catalyzed by Gpx4. Furthermore, excessive glutamate accumulation within the cell can also activate glutamate-NMDA receptors, which in turn promote neuronal iron uptake. These two processes consume antioxidants and result in oxidative stress. The excessive free radicals then target sensitive fatty acids to promote lipid peroxidation, leading to the impaired integrity of lipid membranes and mitochondrial dysfunction, which can release ROS into the cytoplasm. BBB, blood-brain barrier; Gpx4, glutathione peroxidase 4; GSH, glutathione; GSSG, glutathione disulfide; NMDA, N-methyl-D-aspartate; ROS, reactive oxygen species.

confirm the important role of iron overload in ischemic stroke.

\section{Depletion of GSH and Gpx4 Inactivation}

Glutathione is a tripeptide (Glu-Cyc-Gly) that can combine with free radicals to protect cells from oxidative damage (Aoyama and Nakaki, 2015). Gpx4 is an important antioxidant enzyme that converts GSH into oxidized glutathione (GSSG), and then transforms the cytotoxic lipid peroxides to the corresponding alcohols. During ferroptosis, accumulation of redox-active iron consumes GSH reserves through the Fenton reaction, which then suppresses the activity of Gpx4 and leads to an overwhelming antioxidant response (Nunez et al., 2012). The absence of antioxidant enzymes in turn result in the accumulation of iron. In a mouse model of ischemic stroke, decreased GSH and Gpx4 activity in neurons have been identified, accompanied with enhanced lipid peroxidation (Ahmad et al., 2014). A study using brain cells also indicated that a reduction of GSH can sensitize cells to oxidative stress and trigger lipid peroxidation (Andersen et al., 1996). In a gerbil cerebral ischemia model, carvacrol also successfully protects hippocampal neurons against ferroptotic cell death by increasing the expression of $\mathrm{Gpx} 4$, which provides a promising target for ischemic stroke therapies (Guan et al., 2019).

\section{Lipid Peroxidation}

The depletion of GSH, as well as Gpx4 inactivation, has been confirmed as a requisite factor for the promotion of lipid peroxidation during ferroptosis. Specifically, when the antioxidant system is overwhelmed due to the iron overload, excessive free radicals target sensitive fatty acids and promote their peroxidation, which then impairs the integrity of lipid membranes and induces suicide signaling cascades (Reed, 2011; Yin et al., 2011). Besides, it also causes lysosomal membrane permeabilization and the release of redox-active iron into the cytoplasm, which in turn promotes the generation of Fenton radicals, cell membrane denaturation, and GSH depletion (Galluzzi et al., 2014). During this step, peroxidation of polyunsaturated fatty acids (PUFAs) has been considered as a key player (Kagan et al., 2017). Two enzymes, acylCoA synthetase long chain family member 4 (ACSL4) and lysophosphatidylcholine Acyltransferase 3 (LPCAT3), have been identified to be responsible for the biosynthesis and remodeling of PUFA-containing phospholipid, while inhibiting ACSL4 or LPCAT3 prevents ferroptotic cell death (Yuan et al., 2016; Li et al., 2019; Cheng et al., 2020). Then, oxidation of PUFAphosphatidylethanolamines by cyclooxygenases, lipoxygenases (LOX) and cytochromes P450 lead to the accumulation of peroxides, finally contributing to the generation of lipid peroxides (Yang et al., 2016; Çolakoğlu et al., 2018; Tyurina et al., 2018). So far, inhibiting lipid peroxidation through lipoxygenase inhibitors or lipophilic antioxidants has been shown successfully reduce ferroptotic cell death (Matsushita et al., 2015). Since membrane phospholipids in the brain are highly enriched in PUFAs, they are easily disrupted by a high quantity of ROS, and thereby induce lipid peroxidation (Chen et al., 2008). In animal and human ischemic stroke models, it has been found that lipoxygenase inhibitors can play protective roles by eliminating the overexpression of 
lipoxygenases (Cui et al., 2010; Karatas et al., 2018). Besides, the increased 12/15-LOX was also observed following ischemic stroke, contributing to neurological damage, while 12/15-LOX inhibition reversed the detrimental effects (Pekcec et al., 2013; Yigitkanli et al., 2013).

\section{Impaired System Xc-}

Glutamate-induced neurotoxicity is a well-known important mechanism underlying ischemic stroke. In recent years, it was also identified to participate in the process of ferroptosis (Dixon et al., 2012). Non-synaptic extracellular glutamate in the brain is mainly derived from the system Xc-, which is responsible for glutamate extracellular export and cystine import (Domercq et al., 2007). In physiological conditions, system Xc- could maintain a reducing extracellular environment, but in ischemic stroke, excessive glutamate inhibits cystine uptake via inhibiting system Xc- (Banjac et al., 2008), which impairs cystine absorption and decreases the generation of antioxidant GSH (Conrad and Sato, 2012). In addition, the accumulated glutamate within the cell also results in the activation of glutamate-NMDA receptors, which promote neuronal iron uptake and eventually cause ferroptotic damage (Cheah et al., 2006). In neuronal cell lines, it has been shown that both the 5 -LOX inhibitor zileuton and the iron chelator ferrostatin-1 can protect neurons from glutamateinduced oxidative stress, through the inhibition of ferroptosis (Liu et al., 2015).

\section{CROSSTALK BETWEEN AUTOPHAGY AND FERROPTOSIS}

In recent years, emerging studies have identified that some selective autophagy can also degrade damaged mitochondria, aggregated proteins, excess peroxisomes and invading pathogens through recognizing specific cargos, thus allowing the maintenance of intracellular homeostasis (Kraft et al., 2009; Rogov et al., 2014; Khaminets et al., 2016). These autophagic degradation may contribute to iron overload or lipid peroxidation, eventually causing ferroptosis (Kang and Tang, 2017; Zhou et al., 2019; Liu et al., 2020). Since the selective autophagy and ferroptosis have been found to play important roles in ischemic stroke, it is possible that autophagy may participate in the modulation of brain iron accumulation and lipid peroxidation following ischemic process, subsequently promoting ferroptotic cell death. A specific introduction to this hypothesis is identified in Figure 2.

\section{Nuclear Receptor Coactivator 4 (NCOA4)-Mediated Ferritinophagy and Regulation of TfR1}

Iron is necessary for the high metabolic demands of brain cells, however, excessive free iron can promote oxidative stress and cause brain damage; thus, iron availability must be strictly controlled within brain cells. In general, cellular iron is stored in the non-toxic and bioavailable form of ferritin, which is composed of ferritin heavy chains (FTH) and ferritin light chains (FTL) (Arosio et al., 2009), which can prevent harmful oxidative stress when free iron is overloaded, and release iron when cells require it. The primary pathway to release iron from ferritin is via ferritinophagy, which is NCOA4-mediated ferritin degradation. Specifically, NCOA4 links ferritin to growing autophagosomal membranes, where ferritin is degraded and releases chelated iron, which is then used by the cell to induce oxidative stress (Mancias et al., 2014). Several studies have demonstrated that ferritin degradation and cellular free iron

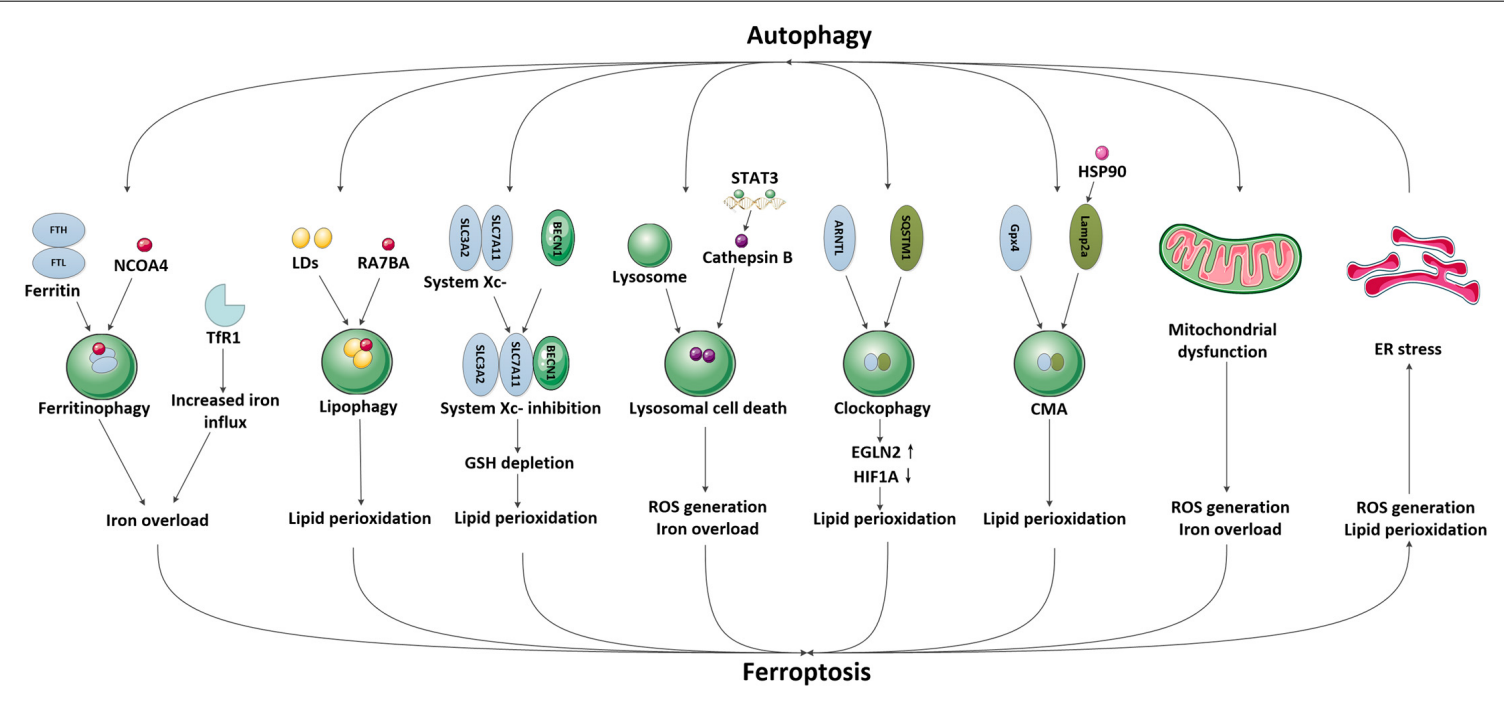

FIGURE 2 | The possible pathways mediating the crosstalk between autophagy and ferroptosis in ischemic stroke. We have illustrated the possible pathways involved in the crosstalk between autophagy and ferroptosis and their downstream effects in ischemic stroke, including NCOA4-mediated ferritinophagy and upregulation of TfR1, RAB7A-mediated lipophagy, BECN1-mediated system Xc- inhibition, STAT3-mediated lysosomal cell death, SQSTM1-mediated clockophagy, HSP90-mediated CMA, mitochondrial dysfunction and ER stress. 
overload, induced by the overexpression of NCOA4, can promote ferroptosis, whereas the depletion or inhibition of NCOA4 increases serum ferritin levels and reduces free iron levels, which thereby inhibits oxidative stress during ferroptosis (Mancias et al., 2015; Gao et al., 2016; Hou et al., 2016). Moreover, the level of GSH is also increased with NCOA4 knockdown and is decreased with NCOA4 overactivation. These results strongly support the presence of a connection between NCOA4-induced ferritinophagy and ferroptosis through the regulation of iron homeostasis and GSH levels.

To date, little is known about the expression of NCOA4 in the human brain, however, it's expression in murine and rat brains has been identified (Siriett et al., 2006; Kollara and Brown, 2010). Acute systemic consumption of NCOA4 shows accumulation of FTH1 within a week, which suggest a potential role of NCOA4 for FTH1 turnover in the brain (Santana-Codina et al., 2019). Interestingly, excessive iron measured as serum ferritin was also associated with poor prognosis following ischemic stroke (García-Yébenes et al., 2012), indicating the important role of ferritin as an iron carrier to mediate iron storage and release under ischemic conditions. Nowadays, a possible link between neurodegeneration and NCOA4-mediated ferritinophagy have been exhaustively reviewed (Quiles Del Rey and Mancias, 2019), however, their effects in ischemic stroke are still unclear. Since the disruption of iron homeostasis is concurrently observed with autophagy defects in ischemic stroke, and autophagy maintains cell homeostasis mainly by promoting the clearance of toxic proteins, we propose that it is highly possible for aberrant autophagy to mediate ferritin degradation, which then promote iron overload and ferroptosis in ischemic stroke.

Transferrin receptor 1 (TfR1) is a major receptor involved in iron transport to the brain, which plays an important role in maintaining the homeostasis of brain iron and regulating ferroptosis (Lo et al., 2007; Raje et al., 2007; Kawabata, 2019). The regulation of TfR 1 expression as well as its related TfR/ Tf endocytic pathway has been identified as a critical event that influences the outcome of ischemic stroke (Lo et al., 2007; Park et al., 2011; Lan et al., 2020). Interestingly, recent studies have shown that activation of autophagy increases the expression of TfR 1 and subsequent intracellular iron (Qian et al., 2002). In wild-type cells, autophagy leads to ferroptosis via the degradation of ferritin and enhanced expression of TfR 1 , while in autophagy-deficient cells and autophagy inhibitor-treated wildtype cells, this effect is abolished (Park and Chung, 2019). These results raise the possibility that autophagy may regulate ischemic stroke by influencing the expression of TfR 1 and irondependent ferroptosis.

\section{RAB7A-Mediated Lipophagy}

Neutral lipids can deposit in the bilayer membranes of the endoplasmic reticulum (ER), which causes the outer layer to expand and form a unique dynamic organelle called lipid droplets (LDs) (Liu and Czaja, 2013). LDs have been found in most eukaryotic cells and in some prokaryotes, and can regulate cellular lipid storage and release in response to metabolic changes (Welte and Gould, 2017). Recently, the autophagic degradation of LDs, also known as lipophagy, has been identified to promote RSL3-induced lipid peroxidation and ferroptosis, while genetically increasing lipid storage by upregulating the level of tumor protein D52 (TPD52) suppressed lipid peroxidation and subsequent ferroptosis (Bai et al., 2019). In rat ischemicreperfusion (I/R) models, studies have found a reshaping of neutral lipids and generation of LDs alongside the induction of lipophagy, which leads to lipid degradation (Lonati et al., 2019). Thus, it is possible that in ischemic stroke, the activated lipophagy might promote lipid peroxidation, which then contributes to ferroptosis.

\section{BECN1-Mediated System Xc- Inhibition}

System Xc- is an amino acid anti-transporter composed of two core components: solute carrier family 7 member 11 (SLC7A11) and solute carrier family 3 member 2 (SLC3A2). It can exchange cystine and glutamate in and out of the cell; the imported cystine then reduces to cysteine and participates in the synthesis of the antioxidant GSH. So far, system Xc- has been shown to be involved in ischemic stroke through modulating glutamate transport and GSH synthesis (Krzyzanowska et al., 2016). In middle cerebral artery occlusion rat models, naotaifang extract treatment significantly inhibits ferroptosis by increasing the SLC7A11/GPX4 pathway (Lan et al., 2020). BECN1 is widely identified as an important autophagy modulator in ischemic stroke (Wang et al., 2014a,b; Liu et al., 2016). Recently, a study reported that BECN1 can also block the activity of system Xc- by directly binding to its core component, SLC7A11, and thereby promoting ferroptosis. In contrast, knockdown of BECN1, or inhibiting the phosphorylation of BECN1 limits the formation of a BECN1-system Xc- complex, which then suppresses ferroptosis induced by system Xc- inhibitors (Song et al., 2018). In primary oligodendrocytes, it has been shown that treatment with glutamate blocks system Xc- function, induces mitochondrial dysfunction, and promotes ferroptosis (Novgorodov et al., 2018). Besides, pretreatment with selenium in neurons attenuates glutamate toxicity, reduces ROS production, and preserves mitochondrial function after glutamate exposure and/or hypoxia, accompanied by reduced levels of BECN1 and LC3-II (Mehta et al., 2012). Since the dysfunction of system $\mathrm{Xc}$ - and BECN1 were concurrently observed with glutamate exposure, we propose whether BECN1 can interact with system $\mathrm{Xc}$ - to regulate ferroptosis in the brain. However, the upstream mechanisms to regulate BECN1 to determine its preferred interaction with system Xc- or Class III PI3K to mediate ferroptosis or autophagy remain undefined, which may be a key topic for future research.

\section{STAT3-Mediated Lysosomal Cell Death}

Autophagy has been confirmed to result in the delivery of cytoplasmic contents and organelles to lysosomes for degradation. Recently, studies have found that lysosomal activity can also be impaired by ferroptosis, which provides a new hypothesis for the relationship between autophagy and ferroptosis. Since lysosomes are vulnerable to oxidative stress, they could be damaged by the intralysosomal Fenton reaction and the subsequent peroxidative instability of lysosomal membranes (Brunk et al., 1995; Ollinger and Brunk, 1995). In contrast, 
intralysosomal ferritin increases lysosomal stability via iron chelation, and then reduces oxidative stress (Garner et al., 1997, 1998). Besides, treatment with lysosome inhibitors has also shown to decrease erastin- and RSL3-induced ferroptotic cell death by inhibiting ROS production and intracellular iron overload (Torii et al., 2016). Signal transducer and activator of transcription 3 (STAT3) is a signaling molecule response to many cytokines and growth factors. It was recently identified that STAT3-mediated overexpression of cathepsin B significantly promotes ferroptosis via the activation of lysosomal cell death, whereas pharmacologically or genetically inhibiting STAT3 blocked ferroptotic cell death (Gao et al., 2018). These findings suggest a potential role of autophagy in ferroptosis via regulation of the lysosomal pathway. Nowadays, increased active cathepsin $B$ levels and lysosomal membrane permeability have been proved to play important roles in ischemic stroke, while treatments reversing these impairments attenuated ischemic damage ( $\mathrm{Ni}$ et al., 2018). However, the relationship between impaired lysosomal activity and ferroptotic damages in ischemic stroke requires further exploration.

\section{SQSTM1-Mediated Clockophagy}

The circadian rhythm is an endogenous oscillation with a periodicity of about $24 \mathrm{~h}$ that is mainly regulated by circadian clock proteins, including aryl hydrocarbon receptor nuclear translocator-like protein 1/brain and muscle ARNT-like 1 (ARNTL/BMAL1) (Partch et al., 2014). It has been shown that the circadian rhythm plays an important role in maintaining normal internal cycles of behavior and brain physiology in human bodies, while its disruption can cause negative effects and even lead to vascular diseases, including ischemic stroke (Karatsoreos et al., 2011). In mouse $I / R$ models, nighttime $I / R$ injury has been found to cause less severe neuronal damage, which is related to the increased expression of circadian proteins such as BMAL1, PERI, and clock proteins (Beker et al., 2018). In human subjects, the outcome of ischemic stroke also shows a diurnal variation through the regulation of circadian clock proteins (Pardiwalla et al., 1993; Elliott, 1998). Besides, the autophagic machinery is inhibited in PER1 $1^{-/-}$hippocampal neurons, which may lead to vulnerability during cerebral ischemia, suggesting a functional relationship between autophagy and circadian rhythm (Rami et al., 2017).

In recent years, emerging studies have suggested that the circadian rhythm could control various cellular processes, including iron metabolism, oxidative stress, and cell death, which indicates its potential role in regulating ferroptosis (Magnone et al., 2014). Besides, the expression of circadian proteins also seems to be modulated by autophagy. A novel type of selective autophagy called clockophagy has been discovered, which is responsible for the degradation of the circadian clock protein ARNTL/BMAL1 via the cargo receptor SQSTM1/p62 (Yang et al., 2019). Early studies have reported that suppressing ARNTL expression by clockophagy effectively contributed to lipid peroxidation and ferroptotic cell death via upregulating the transcription of egl-9 family hypoxia-inducible factor 2 (EGLN2) and then decreasing hypoxia inducible factor 1 alpha (HIF1A)-dependent lipid storage. Genetically or chemically inhibiting ARNTL degradation or EGLN2 activation reduced ferroptosis, whereas destabilizing HIF1A promoted ferroptosis (Yang et al., 2019). These results provide a novel thought for the crosstalk between autophagy and ferroptosis through modulating circadian clock proteins, and prompts the possibility that the decreased expression of circadian clock proteins may lead to a poor prognosis in ischemic stroke due to clockophagyinduced ferroptosis.

\section{HSP90-Mediated CMA}

Chaperone-mediated autophagy (CMA) is responsible for delivering certain cytosolic proteins with a pentapeptide CMAtargeting motif to lysosomes for degradation using molecular chaperones such as HSC70 (heat shock cognate protein 70) and LAMP2a (lysosome-associated membrane protein type 2a). In recent studies, scientists have found that CMA is highly activated during oxidative stress (Kiffin et al., 2004), which enhances the degradation of antioxidant proteins such as Gpx4 and then promote ferroptosis, while inhibition of CMA stabilized Gpx4 and protected against ferroptosis (Shimada et al., 2016; Muller et al., 2017; Zhu et al., 2017). Furthermore, a widely expressed heat shock protein, heat shock protein 90 (HSP90), which can be activated under oxidative stress (Carper et al., 1987; Sidera and Patsavoudi, 2014), has also been identified as an important molecular chaperone that can increase the levels of LAMP2a in the CMA pathway and mediate the degradation of Gpx4 during ferroptosis, while inhibition or knockdown of HSP90 blocks CMA and suppresses ferroptosis in HT-22 cells (a mouse neuronal cell line) (Wu et al., 2019). Since oxidative stress is a critical event during ischemic stroke, we assume that CMA might be activated under ischemic stroke, which then participates in ferroptosis by inducing Gpx4 degradation.

\section{Mitochondrial Dysfunction}

Mitochondrial dysfunction is a major pathological process and also a critical therapeutic target in ischemic stroke. It is regulated by a complex machinery network, which forms a vicious cycle to disrupt mitochondrial homeostasis (Yang J. L. et al., 2018). Mitophagy is a special type of autophagy that can dictate mitochondrial turnover by degrading damaged mitochondria (Pickles et al., 2018). Recently, the activation of mitophagy has been shown to protect against mitochondrial damage in ischemic stroke, which indicates the important role of autophagy in regulating mitochondrial dysfunction (Shen et al., 2017; Yuan et al., 2017). In addition, ferroptosis has also been found to participate in the execution of mitochondrial dysfunction. Morphologically, ferroptosis is characterized by the atrophy of mitochondria with increased membrane densities, as well as reduced or even absent mitochondria crista and a ruptured outer membrane. Mechanically, as a core organelle to regulate iron metabolism, as well as substance and energy metabolism, the impairment of mitochondria can also affect cellular iron utilization and disrupt redox homeostasis, and then contribute to ferroptosis (Li et al., 2020; Wang et al., 2020). In neuronal cells, recent studies have reported that erastin- or RSL3-induced ferroptosis are associated with BID transactivation to mitochondria, increased mitochondrial fragmentation, and 
decreased ATP levels, while the inhibition of BID preserves integrity and function of the mitochondria and prevents ferroptosis (Neitemeier et al., 2017; Jelinek et al., 2018). Since the severity of ferroptosis is tightly associated with the disruption and recovery of mitochondria, while mitophagy is responsible for removing the damaged mitochondria and dictating mitochondrial turnover, we assume that the induction of mitophagy might be able to manipulate ferroptosis via regulating mitochondrial function. Notably, these findings also provide novel concepts regarding therapeutic interventions for ischemic stroke.

\section{ER Stress}

The accumulation of misfolded proteins in ER, known as the unfolded protein response (UPR), disrupts ER homeostasis and leads to ER stress. Nowadays, ER stress has been confirmed as an essential factor mediating cell death in ischemic stroke (Nakka et al., 2010). Autophagy is a critical process activated by ER stress, which is responsible for removing misfolded proteins. In ischemic stroke, the induction of autophagy by ER stress can lead to two-sided effects. On the one hand, inhibition of ER stressdependent autophagy could alleviate acute neuronal ischemic injury (Feng et al., 2017). On the other hand, salubrinal, an ER stress inhibitor, inhibits both the activation of autophagy and neuroprotection mediated by brain ischemic preconditioning (Gao et al., 2013). In contrast, activation of autophagy can also regulate ER stress. It has been shown that inhibition of autophagy by 3-Methyladenine (3-MA) significantly aggravates ER stress in ischemic stroke, while treatment with the autophagy inducer rapamycin reverses these effects (Sheng et al., 2012; Fan et al., 2016).

Recently, emerging studies have also identified the relationship between ER stress and ferroptosis (Dixon et al., 2014). For example, redox imbalance and lipid peroxidation can trigger ER stress (Vladykovskaya et al., 2012). RNA sequencing demonstrated that inhibition of system Xccan lead to the activation of ER stress and upregulation of $\mathrm{CHAC1}$ (ChaC, cation transport regulator homolog 1) (Dixon et al., 2014). Interestingly, ferroptosis could also share cell death pathways with autophagy via the ER stress response. Both ferroptosis inducers [artesunate (ART) and erastin (ERA)] and autophagy inducers [bortezomib (BOR) and XIE62-1004] promote the formation of autophagosome by regulating ER stress (Lee et al., 2020). These results not only provide a better understanding for the manipulation of ER stress, but also provide a new thought for the relationship between autophagy and ferroptosis in ischemic stroke.

\section{Other Potential Pathways Linking Autophagy and Ferroptosis}

In addition to the above, there are some other signals that also indicate potential links between ferroptosis and autophagy. For example, autophagy can significantly decrease the levels of GSH (Mancilla et al., 2015; Stockwell et al., 2017), while both autophagy inhibitors and selective ferroptosis inhibitors improve GSH levels and suppress cell death, and vice versa (Desideri et al., 2012; Sun et al., 2018). These results suggest an important role of GSH in regulating both ferroptosis and autophagy, and that activation of one process might promote another through regulating GSH levels. ACSL4 is a critical enzyme involved in arachidonic acid (AA) metabolism and has been discovered to influence the sensitivity to ferroptosis (Yuan et al., 2016; Doll et al., 2017). Interestingly, it has also been identified as a novel activator of the mTOR pathway (Orlando et al., 2015). Since mTOR can protect cells from excess iron and ferroptosis (Baba et al., 2018), it might be a potential target for ACSL4 to modulate ferroptosis sensitivity. On the other hand, the induction of ferroptosis can also influence autophagy. For example, erastin-induced excessive ROS generation can activate autophagy, while overexpression of Gpx4 suppressed ROS-induced autophagy (Garg et al., 2013). Treatment with curcumin caused significant iron deprivation and then induced protective autophagy, while iron supplementation suppressed the occurrence of autophagy (Yang et al., 2017). Furthermore, the products of lipid peroxidation can also inhibit autophagy by activating mTORC1 signaling under ischemic conditions (Ma et al., 2011), or causing lysosomal dysfunction and lipofuscin generation to reduce autophagy activity (Krohne et al., 2010). Heat shock $70 \mathrm{kDa}$ protein 5 (HSPA5) is an important molecular chaperone expressed primarily in the ER. It has been shown to effectively protect against cell death in response to ER stress-induced autophagy (Chang et al., 2019). Besides, a recent study also demonstrated that overexpression of HSPA5 can negatively regulate ferroptosis by limiting Gpx4 degradation and lipid peroxidation (Zhu et al., 2017). In the future, additional studies are needed to clarify whether the two processes mediated by HSPA5 function separately, or in cooperation with each other.

\section{CONCLUSION AND PERSPECTIVES}

Nowadays, a number of cell death pathways have been discovered (Galluzzi et al., 2014), which can cooperate with each other to help maintain organismal homeostasis. Clarification of their molecular mechanisms and crosstalk between each pathway would not only favor a comprehensive understanding of cell death pathways, but also open up new therapeutic approaches for related diseases. In recent years, the interrelationship between autophagy and ferroptosis has attracted more and more attention, which provides a novel concept regarding the regulation of cell death. However, their potential effects underlying ischemic stroke have not yet been discussed. In this review, we briefly summarize current knowledge on the mechanisms of autophagy and ferroptosis, while focusing on the possible pathways that mediate their crosstalk during ischemic stroke. Nevertheless, a lot of questions still existed before its clinical application. For example, the precise mechanisms underlying ferroptosis to govern iron and lipid metabolism in ischemic stroke remain to be explored, and the functional role of the different types of autophagy or the associated autophagy receptors in ferroptosis are still unclear. Besides, what are the effector molecules downstream of the two 
pathways to induce cell death? And can they influence each other and form a feedback loop in ischemic stroke? Moreover, since autophagy plays a dual role in ischemic stroke, it is critical for future interventions to manipulate the intensity of autophagy to find a balance between ferroptosis and autophagy and minimize neurological damages. Lastly, recent research has revealed that inhibition of two and more cell death pathways simultaneously can decrease ischemic stroke damage more significantly than inhibiting a single one (Tian et al., 2018). Therefore, interventions targeting both autophagy and ferroptosis at the same time could actually provide us with new ideas for the future treatment of ischemic stroke. All in all, there is still a long way to go before we fully understand the crosstalk between these two processes in ischemic stroke.

\section{REFERENCES}

Ahmad, S., Elsherbiny, N. M., Haque, R., Khan, M. B., Ishrat, T., Shah, Z. A., et al. (2014). Sesamin attenuates neurotoxicity in mouse model of ischemic brain stroke. Neurotoxicology 45, 100-110. doi: 10.1016/j.neuro.2014.10.002

Alim, I., Caulfield, J. T., Chen, Y., Swarup, V., Geschwind, D. H., Ivanova, E., et al. (2019). Selenium drives a transcriptional adaptive program to block ferroptosis and treat stroke. Cell 177, 1262-1279.e25. doi: 10.1016/j.cell.2019.03.032

Andersen, J. K., Mo, J. Q., Hom, D. G., Lee, F. Y., Harnish, P., Hamill, R. W., et al. (1996). Effect of buthionine sulfoximine, a synthesis inhibitor of the antioxidant glutathione, on the murine nigrostriatal neurons. J. Neurochem. 67, 2164-2171. doi: 10.1046/j.1471-4159.1996.67052164.x

Aoyama, K., and Nakaki, T. (2015). Glutathione in cellular redox homeostasis: association with the excitatory amino acid carrier 1 (EAAC1). Molecules 20, 8742-8758. doi: 10.3390/molecules 20058742

Arosio, P., Ingrassia, R., and Cavadini, P. (2009). Ferritins: a family of molecules for iron storage, antioxidation and more. Biochim. Biophys. Acta 1790, 589-599. doi: 10.1016/j.bbagen.2008.09.004

Baba, Y., Higa, J. K., Shimada, B. K., Horiuchi, K. M., Suhara, T., Kobayashi, M., et al. (2018). Protective effects of the mechanistic target of rapamycin against excess iron and ferroptosis in cardiomyocytes. Am. J. Physiol. Heart Circ. Physiol. 314, H659-H668. doi: 10.1152/ajpheart.00452.2017

Baek, S.-H., Noh, A. R., Kim, K.-A., Akram, M., Shin, Y.-J., Kim, E.-S., et al. (2014). Modulation of mitochondrial function and autophagy mediates carnosine neuroprotection against ischemic brain damage. Stroke 45, 2438-2443. doi: 10.1161/strokeaha.114.005183

Bai, Y., Meng, L., Han, L., Jia, Y., Zhao, Y., Gao, H., et al. (2019). Lipid storage and lipophagy regulates ferroptosis. Biochem. Biophys. Res. Commun. 508, 997-1003. doi: 10.1016/j.bbrc.2018.12.039

Banjac, A., Perisic, T., Sato, H., Seiler, A., Bannai, S., Weiss, N., et al. (2008). The cystine/cysteine cycle: a redox cycle regulating susceptibility versus resistance to cell death. Oncogene 27, 1618-1628. doi: 10.1038/sj.onc.1210796

Behrends, C., Sowa, M. E., Gygi, S. P., and Harper, J. W. (2010). Network organization of the human autophagy system. Nature 466, 68-76. doi: 10.1038/ nature09204

Beker, M. C., Caglayan, B., Yalcin, E., Caglayan, A. B., Turkseven, S., Gurel, B., et al. (2018). Time-of-day dependent neuronal injury after ischemic stroke: implication of circadian clock transcriptional factor bmal1 and survival kinase AKT. Mol. Neurobiol. 55, 2565-2576. doi: 10.1007/s12035-017-0524-4

Brunk, U. T., Zhang, H., Roberg, K., and Ollinger, K. (1995). Lethal hydrogen peroxide toxicity involves lysosomal iron-catalyzed reactions with membrane damage. Redox. Rep. 1, 267-277. doi: 10.1080/13510002.1995.11746997

Bu, Q., Liu, X., Zhu, Y., Liu, Y., and Wang, Y. (2014). w007B protects brain against ischemia-reperfusion injury in rats through inhibiting inflammation, apoptosis and autophagy. Brain Res. 1558, 100-108. doi: 10.1016/j.brainres.2014.02.034

Carbonell, T., and Rama, R. (2007). Iron, oxidative stress and early neurological deterioration in ischemic stroke. Curr. Med. Chem. 14, 857-874. doi: 10.2174/ 092986707780363014

\section{AUTHOR CONTRIBUTIONS}

JL and Z-NG wrote the manuscript. X-LY, SH, YL, and J-XR prepared the figures. YY reviewed and edited the manuscript. All authors contributed to the article and approved the submitted version.

\section{FUNDING}

This work was supported by the National Natural Science Foundation of China (81771243), the Program for JLU Science and Technology Innovative Research Team (2017TD-12), and Jilin Provincial Key Laboratory (20190901005JC) to YY.

Carper, S. W., Duffy, J. J., and Gerner, E. W. (1987). Heat shock proteins in thermotolerance and other cellular processes. Cancer Res. 47, 5249-5255.

Chang, L., Chai, X., Chen, P., Cao, J., Xie, H., and Zhu, J. (2019). miR-181b-5p suppresses starvation-induced cardiomyocyte autophagy by targeting Hspa5. Int. J. Mol. Med. 43, 143-154. doi: 10.3892/ijmm.2018.3988

Cheah, J. H., Kim, S. F., Hester, L. D., Clancy, K. W., Patterson, S. E. III, Papadopoulos, V., et al. (2006). NMDA receptor-nitric oxide transmission mediates neuronal iron homeostasis via the GTPase Dexras1. Neuron 51, 431-440. doi: 10.1016/j.neuron.2006.07.011

Chen, C. T., Green, J. T., Orr, S. K., and Bazinet, R. P. (2008). Regulation of brain polyunsaturated fatty acid uptake and turnover. Prostaglandins Leukot. Essent. Fatty Acids 79, 85-91. doi: 10.1016/j.plefa.2008.09.003

Chen, H., Yoshioka, H., Kim, G. S., Jung, J. E., Okami, N., Sakata, H., et al. (2011). Oxidative stress in ischemic brain damage: mechanisms of cell death and potential molecular targets for neuroprotection. Antioxid. Redox Signal. 14, 1505-1517. doi: 10.1089/ars.2010.3576

Cheng, J., Fan, Y. Q., Liu, B. H., Zhou, H., Wang, J. M., and Chen, Q. X. (2020). ACSL4 suppresses glioma cells proliferation via activating ferroptosis. Oncol. Rep. 43, 147-158. doi: 10.3892/or.2019.7419

Çolakoğlu, M., Tunçer, S., and Banerjee, S. (2018). Emerging cellular functions of the lipid metabolizing enzyme 15-Lipoxygenase-1. Cell Prolif. 51:e12472. doi: $10.1111 /$ cpr.12472

Conrad, M., and Sato, H. (2012). The oxidative stress-inducible cystine/glutamate antiporter, system x (c) (-) : cystine supplier and beyond. Amino Acids 42, 231-246. doi: 10.1007/s00726-011-0867-5

Cui, L., Zhang, X., Yang, R., Liu, L., Wang, L., Li, M., et al. (2010). Baicalein is neuroprotective in rat MCAO model: role of 12/15-lipoxygenase, mitogenactivated protein kinase and cytosolic phospholipase A2. Pharmacol. Biochem. Behav. 96, 469-475. doi: 10.1016/j.pbb.2010.07.007

Dai, S. H., Chen, T., Li, X., Yue, K. Y., Luo, P., Yang, L. K., et al. (2017). Sirt3 confers protection against neuronal ischemia by inducing autophagy: involvement of the AMPK-mTOR pathway. Free Radic. Biol. Med. 108, 345-353. doi: 10.1016/ j.freeradbiomed.2017.04.005

DeGregorio-Rocasolano, N., Martí-Sistac, O., Ponce, J., Castelló-Ruiz, M., Millán, M., Guirao, V., et al. (2018). Iron-loaded transferrin (Tf) is detrimental whereas iron-free $\mathrm{Tf}$ confers protection against brain ischemia by modifying blood $\mathrm{Tf}$ saturation and subsequent neuronal damage. Redox Biol. 15, 143-158. doi: 10.1016/j.redox.2017.11.026

DeGregorio-Rocasolano, N., Martí-Sistac, O., and Gasull, T. (2019). Deciphering the iron side of stroke: neurodegeneration at the crossroads between iron dyshomeostasis, excitotoxicity, and ferroptosis. Front. Neurosci. 13:85. doi: 10. 3389/fnins.2019.00085

Desideri, E., Filomeni, G., and Ciriolo, M. R. (2012). Glutathione participates in the modulation of starvation-induced autophagy in carcinoma cells. Autophagy 8, 1769-1781. doi: 10.4161/auto.22037

Dixon, S. J., Lemberg, K. M., Lamprecht, M. R., Skouta, R., Zaitsev, E. M., Gleason, C. E., et al. (2012). Ferroptosis: an iron-dependent form of nonapoptotic cell death. Cell 149, 1060-1072. doi: 10.1016/j.cell.2012.03.042 
Dixon, S. J., Patel, D. N., Welsch, M., Skouta, R., Lee, E. D., Hayano, M., et al. (2014). Pharmacological inhibition of cystine-glutamate exchange induces endoplasmic reticulum stress and ferroptosis. eLife 3:e02523. doi: 10.7554/eLife. 02523

Doll, S., Proneth, B., Tyurina, Y. Y., Panzilius, E., Kobayashi, S., Ingold, I., et al. (2017). ACSL4 dictates ferroptosis sensitivity by shaping cellular lipid composition. Nat. Chem. Biol. 13, 91-98. doi: 10.1038/nchembio. 2239

Domercq, M., Sánchez-Gómez, M. V., Sherwin, C., Etxebarria, E., Fern, R., and Matute, C. (2007). System xc- and glutamate transporter inhibition mediates microglial toxicity to oligodendrocytes. J. Immunol. 178, 6549-6556. doi: 10. 4049/jimmunol.178.10.6549

Doria, A., Gatto, M., and Punzi, L. (2013). Autophagy in human health and disease. N. Engl. J. Med. 368, 651-662. doi: 10.1056/NEJMc1303158

Duran, A., Amanchy, R., Linares, J. F., Joshi, J., Abu-Baker, S., Porollo, A., et al. (2011). p62 is a key regulator of nutrient sensing in the mTORC1 pathway. Mol. Cell 44, 134-146. doi: 10.1016/j.molcel.2011.06.038

Egan, D. F., Shackelford, D. B., Mihaylova, M. M., Gelino, S., Kohnz, R. A., Mair, W., et al. (2011). Phosphorylation of ULK1 (hATG1) by AMP-activated protein kinase connects energy sensing to mitophagy. Science 331, 456-461. doi: 10.1126/science.1196371

Elliott, W. J. (1998). Circadian variation in the timing of stroke onset: a metaanalysis. Stroke 29, 992-996. doi: 10.1161/01.str.29.5.992

Fan, T., Huang, Z., Chen, L., Wang, W., Zhang, B., Xu, Y., et al. (2016). Associations between autophagy, the ubiquitin-proteasome system and endoplasmic reticulum stress in hypoxia-deoxygenation or ischemia-reperfusion. Eur. J. Pharmacol. 791, 157-167. doi: 10.1016/j.ejphar.2016.08.026

Feng, D., Wang, B., Wang, L., Abraham, N., Tao, K., Huang, L., et al. (2017). Preischemia melatonin treatment alleviated acute neuronal injury after ischemic stroke by inhibiting endoplasmic reticulum stress-dependent autophagy via PERK and IRE1 signalings. J. Pineal Res. 62:e12395. doi: 10.1111/jpi. 12395

Fujita, N., Itoh, T., Omori, H., Fukuda, M., Noda, T., and Yoshimori, T. (2008). The Atg16L complex specifies the site of LC3 lipidation for membrane biogenesis in autophagy. Mol. Biol. Cell 19, 2092-2100.

Galluzzi, L., Bravo-San Pedro, J. M., and Kroemer, G. (2014). Organelle-specific initiation of cell death. Nat. Cell Biol. 16, 728-736. doi: 10.1038/ncb3005

Gao, B., Zhang, X. Y., Han, R., Zhang, T. T., Chen, C., Qin, Z. H., et al. (2013). The endoplasmic reticulum stress inhibitor salubrinal inhibits the activation of autophagy and neuroprotection induced by brain ischemic preconditioning. Acta Pharmacol. Sin. 34, 657-666. doi: 10.1038/aps.2013.34

Gao, C., Cai, Y., Zhang, X., Huang, H., Wang, J., Wang, Y., et al. (2015). Ischemic preconditioning mediates neuroprotection against ischemia in mouse Hippocampal CA1 neurons by inducing autophagy. PLoS One 10:e137146. doi: 10.1371/journal.pone.0137146

Gao, H., Bai, Y., Jia, Y., Zhao, Y., Kang, R., Tang, D., et al. (2018). Ferroptosis is a lysosomal cell death process. Biochem. Biophys. Res. Commun. 503, 1550-1556. doi: 10.1016/j.bbrc.2018.07.078

Gao, M., Monian, P., Pan, Q., Zhang, W., Xiang, J., and Jiang, X. (2016). Ferroptosis is an autophagic cell death process. Cell Res. 26, 1021-1032. doi: 10.1038/cr. 2016.95

García-Yébenes, I., García-Culebras, A., Peña-Martínez, C., Fernández-López, D., Díaz-Guzmán, J., Negredo, P., et al. (2018). Iron overload exacerbates the risk of hemorrhagic transformation after tPA (Tissue-Type plasminogen activator) administration in thromboembolic stroke mice. Stroke 49, 2163-2172. doi: 10. 1161/strokeaha.118.021540

García-Yébenes, I., Sobrado, M., Moraga, A., Zarruk, J. G., Romera, V. G., Pradillo, J. M., et al. (2012). Iron overload, measured as serum ferritin, increases brain damage induced by focal ischemia and early reperfusion. Neurochem. Int. 61, 1364-1369. doi: 10.1016/j.neuint.2012.09.014

Garg, A. D., Dudek, A. M., Ferreira, G. B., Verfaillie, T., Vandenabeele, P., Krysko, D. V., et al. (2013). ROS-induced autophagy in cancer cells assists in evasion from determinants of immunogenic cell death. Autophagy 9, 1292-1307. doi: 10.4161/auto. 25399

Garner, B., Li, W., Roberg, K., and Brunk, U. T. (1997). On the cytoprotective role of ferritin in macrophages and its ability to enhance lysosomal stability. Free Radic. Res. 27, 487-500. doi: 10.3109/10715769709065788
Garner, B., Roberg, K., and Brunk, U. T. (1998). Endogenous ferritin protects cells with iron-laden lysosomes against oxidative stress. Free Radic. Res. 29, 103-114. doi: 10.1080/10715769800300121

Guan, X., Li, X., Yang, X., Yan, J., Shi, P., Ba, L., et al. (2019). The neuroprotective effects of carvacrol on ischemia/reperfusion-induced hippocampal neuronal impairment by ferroptosis mitigation. Life Sci 235:116795. doi: 10.1016/j.lfs. 2019.116795

Haley, M. J., Krishnan, S., Burrows, D., de Hoog, L., Thakrar, J., Schiessl, I., et al. (2019). Acute high-fat feeding leads to disruptions in glucose homeostasis and worsens stroke outcome. J. Cereb. Blood Flow Metab. 39, 1026-1037. doi: 10. $1177 / 0271678 \times 17744718$

Hanson, L. R., Roeytenberg, A., Martinez, P. M., Coppes, V. G., Sweet, D. C., Rao, R. J., et al. (2009). Intranasal deferoxamine provides increased brain exposure and significant protection in rat ischemic stroke. J. Pharmacol. Exp. Ther. 330, 679-686. doi: 10.1124/jpet.108.149807

Hawkins, P. T., Anderson, K. E., Davidson, K., and Stephens, L. R. (2006). Signalling through Class I PI3Ks in mammalian cells. Biochem. Soc. Trans. 34(Pt 5), 647-662. doi: 10.1042/bst0340647

Hosokawa, N., Hara, T., Kaizuka, T., Kishi, C., Takamura, A., Miura, Y., et al. (2009). Nutrient-dependent mTORC1 association with the ULK1Atg13-FIP200 complex required for autophagy. Mol. Biol. Cell 20, 1981-1991.

Hou, W., Xie, Y., Song, X., Sun, X., Lotze, M. T., Zeh, H. J., et al. (2016). Autophagy promotes ferroptosis by degradation of ferritin. Autophagy 12, 1425-1428. doi: 10.1080/15548627.2016.1187366

Jelinek, A., Heyder, L., Daude, M., Plessner, M., Krippner, S., Grosse, R., et al. (2018). Mitochondrial rescue prevents glutathione peroxidase-dependent ferroptosis. Free Radic. Biol. Med. 117, 45-57. doi: 10.1016/j.freeradbiomed. 2018.01.019

Jiang, W.-W., Huang, B.-S., Han, Y., Deng, L.-H., and Wu, L.-X. (2017). Sodium hydrosulfide attenuates cerebral ischemia/reperfusion injury by suppressing overactivated autophagy in rats. FEBS Open Bio 7, 1686-1695. doi: 10.1002/ 2211-5463.12301

Kagan, V. E., Mao, G., Qu, F., Angeli, J. P., Doll, S., Croix, C. S., et al. (2017). Oxidized arachidonic and adrenic PEs navigate cells to ferroptosis. Nat. Chem. Biol. 13, 81-90. doi: 10.1038/nchembio.2238

Kang, R., and Tang, D. (2017). Autophagy and Ferroptosis - What's the Connection? Curr. Pathobiol. Rep. 5, 153-159. doi: 10.1007/s40139-017-0139-5

Karatas, H., Eun Jung, J., Lo, E. H., and van Leyen, K. (2018). Inhibiting 12/15lipoxygenase to treat acute stroke in permanent and tPA induced thrombolysis models. Brain Res. 1678, 123-128. doi: 10.1016/j.brainres.2017.10.024

Karatsoreos, I. N., Bhagat, S., Bloss, E. B., Morrison, J. H., and McEwen, B. S. (2011). Disruption of circadian clocks has ramifications for metabolism, brain, and behavior. Proc. Natl. Acad. Sci. U.S.A. 108, 1657-1662. doi: 10.1073/pnas. 1018375108

Kawabata, H. (2019). Transferrin and transferrin receptors update. Free Radic. Biol. Med. 133, 46-54. doi: 10.1016/j.freeradbiomed.2018.06.037

Kell, D. B. (2009). Iron behaving badly: inappropriate iron chelation as a major contributor to the aetiology of vascular and other progressive inflammatory and degenerative diseases. BMC Med. Genomics 2:2. doi: 10.1186/1755-8794-2-2

Khaminets, A., Behl, C., and Dikic, I. (2016). Ubiquitin-dependent and independent signals in selective autophagy. Trends Cell Biol. 26, 6-16. doi: 10.1016/j.tcb.2015.08.010

Kiffin, R., Christian, C., Knecht, E., and Cuervo, A. M. (2004). Activation of chaperone-mediated autophagy during oxidative stress. Mol. Biol. Cell 15, 4829-4840. doi: 10.1091/mbc.e04-06-0477

Kihara, A., Kabeya, Y., Ohsumi, Y., and Yoshimori, T. (2001). Beclinphosphatidylinositol 3-kinase complex functions at the trans-Golgi network. EMBO Rep. 2, 330-335. doi: 10.1093/embo-reports/kve061

Kim, P. K., Hailey, D. W., Mullen, R. T., and Lippincott-Schwartz, J. (2008). Ubiquitin signals autophagic degradation of cytosolic proteins and peroxisomes. Proc. Natl. Acad. Sci. U.S.A. 105, 20567-20574. doi: 10.1073/pnas. 0810611105

Klionsky, D. J., Abdelmohsen, K., Abe, A., Abedin, M. J., Abeliovich, H., Acevedo Arozena, A., et al. (2016). Guidelines for the use and interpretation of assays for monitoring autophagy (3rd edition). Autophagy 12, 1-222. doi: 10.1080/ 15548627.2015.1100356 
Kollara, A., and Brown, T. J. (2010). Variable expression of nuclear receptor coactivator 4 (NcoA4) during mouse embryonic development. J. Histochem. Cytochem. 58, 595-609. doi: 10.1369/jhc.2010.955294

Kraft, C., Reggiori, F., and Peter, M. (2009). Selective types of autophagy in yeast. Biochim. Biophys. Acta 1793, 1404-1412. doi: 10.1016/j.bbamcr.2009.02.006

Krohne, T. U., Kaemmerer, E., Holz, F. G., and Kopitz, J. (2010). Lipid peroxidation products reduce lysosomal protease activities in human retinal pigment epithelial cells via two different mechanisms of action. Exp. Eye Res. 90, 261-266. doi: 10.1016/j.exer.2009.10.014

Krzyzanowska, W., Pomierny, B., Budziszewska, B., Filip, M., and Pera, J. (2016). $\mathrm{N}$-Acetylcysteine and ceftriaxone as preconditioning strategies in focal brain ischemia: influence on glutamate transporters expression. Neurotox. Res. 29, 539-550. doi: 10.1007/s12640-016-9602-z

Lan, B., Ge, J. W., Cheng, S. W., Zheng, X. L., Liao, J., He, C., et al. (2020). Extract of Naotaifang, a compound Chinese herbal medicine, protects neuron ferroptosis induced by acute cerebral ischemia in rats. J. Integr. Med. doi: 10.1016/j.joim. 2020.01.008 [Epub ahead of print].

Lee, Y. S., Kalimuthu, K., Seok Park, Y., Makala, H., Watkins, S. C., Choudry, M. H. A., et al. (2020). Ferroptotic agent-induced endoplasmic reticulum stress response plays a pivotal role in the autophagic process outcome. J. Cell. Physiol. 235, 6767-6778. doi: 10.1002/jcp.29571

Li, J., Cao, F., Yin, H. L., Huang, Z. J., Lin, Z. T., Mao, N., et al. (2020). Ferroptosis: past, present and future. Cell Death Dis. 11:88. doi: 10.1038/s41419-020-2298-2

Li, L., Khatibi, N. H., Hu, Q., Yan, J., Chen, C., Han, J., et al. (2012). Transmembrane protein 166 regulates autophagic and apoptotic activities following focal cerebral ischemic injury in rats. Exp. Neurol. 234, 181-190. doi: 10.1016/j. expneurol.2011.12.038

Li, Q., Zhang, T., Wang, J., Zhang, Z., Zhai, Y., Yang, G. Y., et al. (2014). Rapamycin attenuates mitochondrial dysfunction via activation of mitophagy in experimental ischemic stroke. Biochem. Biophys. Res. Commun. 444, 182188. doi: 10.1016/j.bbrc.2014.01.032

Li, Y., Feng, D., Wang, Z., Zhao, Y., Sun, R., Tian, D., et al. (2019). Ischemiainduced ACSL4 activation contributes to ferroptosis-mediated tissue injury in intestinal ischemia/reperfusion. Cell Death Differ. 26, 2284-2299. doi: 10.1038/ s41418-019-0299-4

Lian, J., Wu, X., He, F., Karnak, D., Tang, W., Meng, Y., et al. (2011). A natural BH3 mimetic induces autophagy in apoptosis-resistant prostate cancer via modulating Bcl-2-Beclin1 interaction at endoplasmic reticulum. Cell Death Differ. 18, 60-71. doi: 10.1038/cdd.2010.74

Liang, X. H., Jackson, S., Seaman, M., Brown, K., Kempkes, B., Hibshoosh, H., et al. (1999). Induction of autophagy and inhibition of tumorigenesis by beclin 1 . Nature 402, 672-676. doi: 10.1038/45257

Liu, J., Kuang, F., Kroemer, G., Klionsky, D. J., Kang, R., and Tang, D. (2020). Autophagy-dependent ferroptosis: machinery and regulation. Cell Chem. Biol. 27, 420-435. doi: 10.1016/j.chembiol.2020.02.005

Liu, K., and Czaja, M. J. (2013). Regulation of lipid stores and metabolism by lipophagy. Cell Death Differ. 20, 3-11. doi: 10.1038/cdd.2012.63

Liu, W., Shang, G., Yang, S., Huang, J., Xue, X., Lin, Y., et al. (2016). Electroacupuncture protects against ischemic stroke by reducing autophagosome formation and inhibiting autophagy through the mTORC1-ULK1 complex-Beclin1 pathway. Int. J. Mol. Med. 37, 309-318. doi: 10.3892/ijmm.2015.2425

Liu, Y., Wang, W., Li, Y., Xiao, Y., Cheng, J., and Jia, J. (2015). The 5-lipoxygenase inhibitor zileuton confers neuroprotection against glutamate oxidative damage by inhibiting ferroptosis. Biol. Pharm. Bull. 38, 1234-1239. doi: 10.1248/bpb. b15-00048

Liu, Y.-J., Wang, D.-Y., Yang, Y.-J., and Lei, W.-F. (2017). Effects and mechanism of dexmedetomidine on neuronal cell injury induced by hypoxia-ischemia. $B M C$ Anesthesiol. 17:117. doi: 10.1186/s12871-017-0413-4

Lo, A. C., Cheung, A. K., Hung, V. K., Yeung, C. M., He, Q. Y., Chiu, J. F., et al. (2007). Deletion of aldose reductase leads to protection against cerebral ischemic injury. J. Cereb. Blood Flow Metab. 27, 1496-1509. doi: 10.1038/sj. jcbfm.9600452

Lonati, E., Corsetto, P. A., Montorfano, G., Zava, S., Carrozzini, T., Brambilla, A., et al. (2019). Lipid reshaping and lipophagy are induced in a modeled ischemia-reperfusion injury of blood brain barrier. Int. J. Mol. Sci. 20:3752. doi: $10.3390 / \mathrm{ijms} 20153752$
Lu, L. Q., Tian, J., Luo, X. J., and Peng, J. (2020). Targeting the pathways of regulated necrosis: a potential strategy for alleviation of cardio-cerebrovascular injury. Cell. Mol. Life Sci. doi: 10.1007/s00018-020-03587-8 [Epub ahead of print].

Luo, C., Ouyang, M. W., Fang, Y. Y., Li, S. J., Zhou, Q., Fan, J., et al. (2017). Dexmedetomidine protects mouse brain from ischemia-reperfusion injury via inhibiting neuronal autophagy through up-regulating HIF-1alpha. Front. Cell. Neurosci. 11:197. doi: 10.3389/fncel.2017.00197

Ma, H., Guo, R., Yu, L., Zhang, Y., and Ren, J. (2011). Aldehyde dehydrogenase 2 (ALDH2) rescues myocardial ischaemia/reperfusion injury: role of autophagy paradox and toxic aldehyde. Eur. Heart J. 32, 1025-1038. doi: 10.1093/ eurheartj/ehq253

Magnone, M. C., Langmesser, S., Bezdek, A. C., Tallone, T., Rusconi, S., and Albrecht, U. (2014). The Mammalian circadian clock gene per2 modulates cell death in response to oxidative stress. Front. Neurol. 5:289. doi: 10.3389/fneur. 2014.00289

Mancias, J. D., Pontano Vaites, L., Nissim, S., Biancur, D. E., Kim, A. J., Wang, X., et al. (2015). Ferritinophagy via NCOA4 is required for erythropoiesis and is regulated by iron dependent HERC2-mediated proteolysis. eLife 4:e10308. doi: 10.7554/eLife.10308

Mancias, J. D., Wang, X., Gygi, S. P., Harper, J. W., and Kimmelman, A. C. (2014). Quantitative proteomics identifies NCOA4 as the cargo receptor mediating ferritinophagy. Nature 509, 105-109. doi: 10.1038/nature13148

Mancilla, H., Maldonado, R., Cereceda, K., Villarroel-Espindola, F., Montes de Oca, M., Angulo, C., et al. (2015). Glutathione Depletion Induces Spermatogonial Cell Autophagy. J. Cell. Biochem. 116, 2283-2292. doi: 10.1002/jcb.25178

Matsushita, M., Freigang, S., Schneider, C., Conrad, M., Bornkamm, G. W., and Kopf, M. (2015). T cell lipid peroxidation induces ferroptosis and prevents immunity to infection. J. Exp. Med. 212, 555-568. doi: 10.1084/jem.20140857

Mehta, S. L., Kumari, S., Mendelev, N., and Li, P. A. (2012). Selenium preserves mitochondrial function, stimulates mitochondrial biogenesis, and reduces infarct volume after focal cerebral ischemia. BMC Neurosci. 13:79. doi: 10.1186/ 1471-2202-13-79

Millan, M., Sobrino, T., Castellanos, M., Nombela, F., Arenillas, J. F., Riva, E., et al. (2007). Increased body iron stores are associated with poor outcome after thrombolytic treatment in acute stroke. Stroke 38, 90-95. doi: 10.1161/01.STR. 0000251798.25803.e0

Mizushima, N., and Komatsu, M. (2011). Autophagy: renovation of cells and tissues. Cell 147, 728-741. doi: 10.1016/j.cell.2011.10.026

Moscat, J., Karin, M., and Diaz-Meco, M. T. (2016). p62 in cancer: signaling adaptor beyond autophagy. Cell 167, 606-609. doi: 10.1016/j.cell.2016.09.030

Muller, T., Dewitz, C., Schmitz, J., Schroder, A. S., Brasen, J. H., Stockwell, B. R., et al. (2017). Necroptosis and ferroptosis are alternative cell death pathways that operate in acute kidney failure. Cell. Mol. Life Sci. 74, 3631-3645. doi: 10.1007/s00018-017-2547-4

Nakatogawa, H. (2013). Two ubiquitin-like conjugation systems that mediate membrane formation during autophagy. Essays Biochem. 55, 39-50. doi: 10. 1042/bse0550039

Nakka, V. P., Gusain, A., and Raghubir, R. (2010). Endoplasmic reticulum stress plays critical role in brain damage after cerebral ischemia/reperfusion in rats. Neurotox. Res. 17, 189-202. doi: 10.1007/s12640-009-9110-5

Neitemeier, S., Jelinek, A., Laino, V., Hoffmann, L., Eisenbach, I., Eying, R., et al. (2017). BID links ferroptosis to mitochondrial cell death pathways. Redox Biol. 12, 558-570. doi: 10.1016/j.redox.2017.03.007

Ni, Y., Gu, W. W., Liu, Z. H., Zhu, Y. M., Rong, J. G., Kent, T. A., et al. (2018). RIP1K contributes to neuronal and astrocytic cell death in ischemic stroke via activating autophagic-lysosomal pathway. Neuroscience 371, 60-74. doi: 10.1016/j.neuroscience.2017.10.038

Novgorodov, S. A., Voltin, J. R., Gooz, M. A., Li, L., Lemasters, J. J., and Gudz, T. I. (2018). Acid sphingomyelinase promotes mitochondrial dysfunction due to glutamate-induced regulated necrosis. J. Lipid Res. 59, 312-329. doi: 10.1194/ jlr.M080374

Nunez, M. T., Urrutia, P., Mena, N., Aguirre, P., Tapia, V., and Salazar, J. (2012). Iron toxicity in neurodegeneration. Biometals 25, 761-776. doi: 10.1007/ s10534-012-9523-0

Ollinger, K., and Brunk, U. T. (1995). Cellular injury induced by oxidative stress is mediated through lysosomal damage. Free Radic. Biol. Med. 19, 565-574. doi: 10.1016/0891-5849(95)00062-3 
Orlando, U. D., Castillo, A. F., Dattilo, M. A., Solano, A. R., Maloberti, P. M., and Podesta, E. J. (2015). Acyl-CoA synthetase-4, a new regulator of mTOR and a potential therapeutic target for enhanced estrogen receptor function in receptor-positive and -negative breast cancer. Oncotarget 6, 42632-42650. doi: 10.18632/oncotarget.5822

Papazisis, G., Pourzitaki, C., Sardeli, C., Lallas, A., Amaniti, E., and Kouvelas, D. (2008). Deferoxamine decreases the excitatory amino acid levels and improves the histological outcome in the hippocampus of neonatal rats after hypoxiaischemia. Pharmacol. Res. 57, 73-77678. doi: 10.1016/j.phrs.2007.12.003

Pardiwalla, F. K., Yeolekar, M. E., and Bakshi, S. K. (1993). Circadian rhythm in acute stroke. J. Assoc. Physicians India 41, 203-204.

Park, E., and Chung, S. W. (2019). ROS-mediated autophagy increases intracellular iron levels and ferroptosis by ferritin and transferrin receptor regulation. Cell Death Dis. 10:822. doi: 10.1038/s41419-019-2064-5

Park, U. J., Lee, Y. A., Won, S. M., Lee, J. H., Kang, S. H., Springer, J. E., et al. (2011). Blood-derived iron mediates free radical production and neuronal death in the hippocampal CA1 area following transient forebrain ischemia in rat. Acta Neuropathol. 121, 459-473. doi: 10.1007/s00401-010-0785-8

Partch, C. L., Green, C. B., and Takahashi, J. S. (2014). Molecular architecture of the mammalian circadian clock. Trends Cell Biol. 24, 90-99. doi: 10.1016/j.tcb. 2013.07.002

Pekcec, A., Yigitkanli, K., Jung, J. E., Pallast, S., Xing, C., Antipenko, A., et al. (2013). Following experimental stroke, the recovering brain is vulnerable to lipoxygenase-dependent semaphorin signaling. FASEB J. 27, 437-445. doi: 10. 1096/fj.12-206896

Pickles, S., Vigié, P., and Youle, R. J. (2018). Mitophagy and quality control mechanisms in mitochondrial maintenance. Curr. Biol. 28, R170-R185. doi: 10.1016/j.cub.2018.01.004

Pilli, M., Arko-Mensah, J., Ponpuak, M., Roberts, E., Master, S., Mandell, M. A., et al. (2012). TBK-1 promotes autophagy-mediated antimicrobial defense by controlling autophagosome maturation. Immunity 37, 223-234. doi: 10.1016/j. immuni.2012.04.015

Qi, Z., Dong, W., Shi, W., Wang, R., Zhang, C., Zhao, Y., et al. (2015). Bcl-2 phosphorylation triggers autophagy switch and reduces mitochondrial damage in limb remote ischemic conditioned rats after ischemic stroke. Transl. Stroke Res. 6, 198-206. doi: 10.1007/s12975-015-0393-y

Qian, Z. M., Li, H., Sun, H., and Ho, K. (2002). Targeted drug delivery via the transferrin receptor-mediated endocytosis pathway. Pharmacol. Rev. 54, 561-587. doi: 10.1124/pr.54.4.561

Quiles Del Rey, M., and Mancias, J. D. (2019). NCOA4-mediated ferritinophagy: a potential link to neurodegeneration. Front. Neurosci. 13:238. doi: 10.3389/fnins. 2019.00238

Raje, C. I., Kumar, S., Harle, A., Nanda, J. S., and Raje, M. (2007). The macrophage cell surface glyceraldehyde-3-phosphate dehydrogenase is a novel transferrin receptor. J. Biol. Chem. 282, 3252-3261. doi: 10.1074/jbc.M60832 8200

Rami, A., Fekadu, J., and Rawashdeh, O. (2017). The Hippocampal autophagic machinery is depressed in the absence of the circadian clock protein PER 1 that may lead to vulnerability during cerebral ischemia. Curr. Neurovasc. Res. 14, 207-214. doi: 10.2174/1567202614666170619083239

Reed, T. T. (2011). Lipid peroxidation and neurodegenerative disease. Free Radic. Biol. Med. 51, 1302-1319. doi: 10.1016/j.freeradbiomed.2011.06.027

Rogov, V., Dötsch, V., Johansen, T., and Kirkin, V. (2014). Interactions between autophagy receptors and ubiquitin-like proteins form the molecular basis for selective autophagy. Mol. Cell 53, 167-178. doi: 10.1016/j.molcel.2013. 12.014

Russell, R. C., Tian, Y., Yuan, H., Park, H. W., Chang, Y. Y., Kim, J., et al. (2013). ULK1 induces autophagy by phosphorylating Beclin-1 and activating VPS34 lipid kinase. Nat. Cell Biol. 15, 741-750. doi: 10.1038/ncb2757

Santana-Codina, N., Gableske, S., Quiles del Rey, M., Małachowska, B., Jedrychowski, M. P., Biancur, D. E., et al. (2019). NCOA4 maintains murine erythropoiesis via cell autonomous and non-autonomous mechanisms. Haematologica 104, 1342-1354. doi: 10.3324/haematol.2018.204123

Shen, Z., Zheng, Y., Wu, J., Chen, Y., Wu, X., Zhou, Y., et al. (2017). PARK2dependent mitophagy induced by acidic postconditioning protects against focal cerebral ischemia and extends the reperfusion window. Autophagy 13, 473-485. doi: 10.1080/15548627.2016.1274596
Sheng, R., Liu, X. Q., Zhang, L. S., Gao, B., Han, R., Wu, Y. Q., et al. (2012). Autophagy regulates endoplasmic reticulum stress in ischemic preconditioning. Autophagy 8, 310-325. doi: 10.4161/auto.18673

Shimada, K., Skouta, R., Kaplan, A., Yang, W. S., Hayano, M., Dixon, S. J., et al. (2016). Global survey of cell death mechanisms reveals metabolic regulation of ferroptosis. Nat. Chem. Biol. 12, 497-503. doi: 10.1038/nchembio.2079

Sidera, K., and Patsavoudi, E. (2014). HSP90 inhibitors: current development and potential in cancer therapy. Recent Pat. Anticancer Drug Discov. 9, 1-20.

Siriett, V., Nicholas, G., Berry, C., Watson, T., Hennebry, A., Thomas, M., et al. (2006). Myostatin negatively regulates the expression of the steroid receptor co-factor ARA70. J. Cell. Physiol. 206, 255-263. doi: 10.1002/jcp.20456

Song, X., Zhu, S., Chen, P., Hou, W., Wen, Q., Liu, J., et al. (2018). AMPK-Mediated BECN1 phosphorylation promotes ferroptosis by directly blocking system Xc(-) activity. Curr. Biol. 28, 2388-2399.e5. doi: 10.1016/j.cub.2018.05.094

Stockwell, B. R., Friedmann Angeli, J. P., Bayir, H., Bush, A. I., Conrad, M., Dixon, S. J., et al. (2017). Ferroptosis: a regulated cell death nexus linking metabolism, redox biology, and disease. Cell 171, 273-285. doi: 10.1016/j.cell.2017.09.021

Sun, Y., Zheng, Y., Wang, C., and Liu, Y. (2018). Glutathione depletion induces ferroptosis, autophagy, and premature cell senescence in retinal pigment epithelial cells. Cell Death Dis. 9:753. doi: 10.1038/s41419-018-0794-4

Thi, E. P., and Reiner, N. E. (2012). Phosphatidylinositol 3-kinases and their roles in phagosome maturation. J. Leukoc. Biol. 92, 553-566. doi: 10.1189/jlb.0212053

Tian, J., Guo, S., Chen, H., Peng, J. J., Jia, M. M., Li, N. S., et al. (2018). Combination of emricasan with ponatinib synergistically reduces ischemia/reperfusion injury in rat brain through simultaneous prevention of apoptosis and necroptosis. Transl. Stroke Res. 9, 382-392. doi: 10.1007/s12975-017-0581-z

Torii, S., Shintoku, R., Kubota, C., Yaegashi, M., Torii, R., Sasaki, M., et al. (2016). An essential role for functional lysosomes in ferroptosis of cancer cells. Biochem J 473, 769-777. doi: 10.1042/bj20150658

Tuo, Q. Z., Lei, P., Jackman, K. A., Li, X. L., Xiong, H., Li, X. L., et al. (2017). Taumediated iron export prevents ferroptotic damage after ischemic stroke. Mol. Psychiatry 22, 1520-1530. doi: 10.1038/mp.2017.171

Tyurina, Y. Y., Shrivastava, I., Tyurin, V. A., Mao, G., Dar, H. H., Watkins, S., et al. (2018). "Only a life lived for others is worth living": redox signaling by oxygenated phospholipids in cell fate decisions. Antioxid. Redox Signal. 29, 1333-1358. doi: 10.1089/ars.2017.7124

Umemura, A., He, F., Taniguchi, K., Nakagawa, H., Yamachika, S., Font-Burgada, J., et al. (2016). p62, upregulated during preneoplasia, induces hepatocellular carcinogenesis by maintaining survival of stressed HCC-initiating cells. Cancer Cell 29, 935-948. doi: 10.1016/j.ccell.2016.04.006

van Leyen, K., Kim, H. Y., Lee, S.-R., Jin, G., Arai, K., and Lo, E. H. (2006). Baicalein and 12/15-lipoxygenase in the ischemic brain. Stroke 37, 3014-3018. doi: 10.1161/01.STR.0000249004.25444.a5

Vladykovskaya, E., Sithu, S. D., Haberzettl, P., Wickramasinghe, N. S., Merchant, M. L., Hill, B. G., et al. (2012). Lipid peroxidation product 4-hydroxy-trans2-nonenal causes endothelial activation by inducing endoplasmic reticulum stress. J. Biol. Chem. 287, 11398-11409. doi: 10.1074/jbc.M111.320416

Wang, H., Liu, C., Zhao, Y., and Gao, G. (2020). Mitochondria regulation in ferroptosis. Eur. J. Cell Biol. 99:151058. doi: 10.1016/j.ejcb.2019.151058

Wang, N., Yang, L., Zhang, H., Lu, X., Wang, J., Cao, Y., et al. (2018). MicroRNA$9 a-5 p$ alleviates ischemia injury after focal cerebral ischemia of the rat by targeting ATG5-mediated autophagy. Cell. Physiol. Biochem. 45, 78-87. doi: 10.1159/000486224

Wang, P., Liang, J., Li, Y., Li, J., Yang, X., Zhang, X., et al. (2014a). Down-regulation of miRNA-30a alleviates cerebral ischemic injury through enhancing beclin 1mediated autophagy. Neurochem. Res. 39, 1279-1291. doi: 10.1007/s11064-0141310-6

Wang, P., Shao, B. Z., Deng, Z., Chen, S., Yue, Z., and Miao, C. Y. (2018). Autophagy in ischemic stroke. Prog. Neurobiol. 163-164, 98-117. doi: 10.1016/ j.pneurobio.2018.01.001

Wang, P., Xu, T. Y., Wei, K., Guan, Y. F., Wang, X., Xu, H., et al. (2014b). ARRB1/ $\beta$-arrestin-1 mediates neuroprotection through coordination of BECN1-dependent autophagy in cerebral ischemia. Autophagy 10, 15351548. doi: 10.4161/auto.29203

Wang, S., Han, X., Mao, Z., Xin, Y., Maharjan, S., and Zhang, B. (2019). MALAT1 lncRNA induces autophagy and protects brain microvascular endothelial cells against oxygen-glucose deprivation by binding to miR-200c-3p and 
upregulating SIRT1 expression. Neuroscience 397, 116-126. doi: 10.1016/j. neuroscience.2018.11.024

Wang, S., Li, J., Du, Y., Xu, Y., Wang, Y., Zhang, Z., et al. (2017). The Class I PI3K inhibitor S14161 induces autophagy in malignant blood cells by modulating the Beclin 1/Vps34 complex. J. Pharmacol. Sci. 134, 197-202. doi: 10.1016/j.jphs. 2017.07.001

Welte, M. A., and Gould, A. P. (2017). Lipid droplet functions beyond energy storage. Biochim. Biophys. Acta 1862(10 Pt B), 1260-1272. doi: 10.1016/j.bbalip. 2017.07.006

Wu, Z., Geng, Y., Lu, X., Shi, Y., Wu, G., Zhang, M., et al. (2019). Chaperonemediated autophagy is involved in the execution of ferroptosis. Proc. Natl. Acad. Sci. U.S.A. 116, 2996-3005. doi: 10.1073/pnas.1819728116

Xie, Y., Hou, W., Song, X., Yu, Y., Huang, J., Sun, X., et al. (2016). Ferroptosis: process and function. Cell Death Differ. 23, 369-379. doi: 10.1038/cdd.2015.158

Xie, Y., Kang, R., Sun, X., Zhong, M., Huang, J., Klionsky, D. J., et al. (2015). Posttranslational modification of autophagy-related proteins in macroautophagy. Autophagy 11, 28-45. doi: 10.4161/15548627.2014.984267

Yang, C., Ma, X., Wang, Z., Zeng, X., Hu, Z., Ye, Z., et al. (2017). Curcumin induces apoptosis and protective autophagy in castration-resistant prostate cancer cells through iron chelation. Drug Des. Dev. Ther. 11, 431-439. doi: 10.2147/dddt. s126964

Yang, G., Wang, N., Seto, S. W., Chang, D., and Liang, H. (2018). Hydroxysafflor yellow a protects brain microvascular endothelial cells against oxygen glucose deprivation/reoxygenation injury: involvement of inhibiting autophagy via class I PI3K/Akt/mTOR signaling pathway. Brain Res. Bull. 140, 243-257. doi: 10.1016/j.brainresbull.2018.05.011

Yang, J., and Yao, S. (2015). JNK-Bcl-2/Bcl-xL-Bax/Bak Pathway Mediates the Crosstalk between Matrine-Induced Autophagy and Apoptosis via Interplay with Beclin 1. Int. J. Mol. Sci. 16, 25744-25758. doi: 10.3390/ijms161025744

Yang, J. L., Mukda, S., and Chen, S. D. (2018). Diverse roles of mitochondria in ischemic stroke. Redox Biol. 16, 263-275. doi: 10.1016/j.redox.2018.03.002

Yang, M., Chen, P., Liu, J., Zhu, S., Kroemer, G., Klionsky, D. J., et al. (2019). Clockophagy is a novel selective autophagy process favoring ferroptosis. Sci. Adv. 5:eaaw2238. doi: 10.1126/sciadv.aaw2238

Yang, W. S., Kim, K. J., Gaschler, M. M., Patel, M., Shchepinov, M. S., and Stockwell, B. R. (2016). Peroxidation of polyunsaturated fatty acids by lipoxygenases drives ferroptosis. Proc. Natl. Acad. Sci. U.S.A. 113, E4966-E4975. doi: 10.1073/pnas. 1603244113

Yao, F., Lv, Y. C., Zhang, M., Xie, W., Tan, Y. L., Gong, D., et al. (2015). Apelin13 impedes foam cell formation by activating Class III PI3K/Beclin-1-mediated autophagic pathway. Biochem. Biophys. Res. Commun. 466, 637-643. doi: 10. 1016/j.bbrc.2015.09.045
Yigitkanli, K., Pekcec, A., Karatas, H., Pallast, S., Mandeville, E., Joshi, N., et al. (2013). Inhibition of 12/15-lipoxygenase as therapeutic strategy to treat stroke. Ann. Neurol. 73, 129-135. doi: 10.1002/ana.23734

Yin, H., Xu, L., and Porter, N. A. (2011). Free radical lipid peroxidation: mechanisms and analysis. Chem. Rev. 111, 5944-5972. doi: 10.1021/cr200 $084 \mathrm{z}$

Yu, S., Yu, M., He, X., Wen, L., Bu, Z., and Feng, J. (2019). KCNQ1OT1 promotes autophagy by regulating miR-200a/FOXO3/ATG7 pathway in cerebral ischemic stroke. Aging Cell 18:e12940. doi: 10.1111/acel.12940

Yuan, H., Li, X., Zhang, X., Kang, R., and Tang, D. (2016). Identification of ACSL4 as a biomarker and contributor of ferroptosis. Biochem. Biophys. Res. Commun. 478, 1338-1343. doi: 10.1016/j.bbrc.2016.08.124

Yuan, Y., Zheng, Y., Zhang, X., Chen, Y., Wu, X., Wu, J., et al. (2017). BNIP3L/NIXmediated mitophagy protects against ischemic brain injury independent of PARK2. Autophagy 13, 1754-1766. doi: 10.1080/15548627.2017.135 7792

Zhang, X., Yuan, Y., Jiang, L., Zhang, J., Gao, J., Shen, Z., et al. (2014). Endoplasmic reticulum stress induced by tunicamycin and thapsigargin protects against transient ischemic brain injury: involvement of PARK2-dependent mitophagy. Autophagy 10, 1801-1813. doi: 10.4161/auto.32136

Zhou, B., Liu, J., Kang, R., Klionsky, D. J., Kroemer, G., and Tang, D. (2019). Ferroptosis is a type of autophagy-dependent cell death. Semin. Cancer Biol. doi: 10.1016/j.semcancer.2019.03.002 [Epub ahead of print].

Zhu, S., Zhang, Q., Sun, X., Zeh, H. J. III, Lotze, M. T., Kang, R., et al. (2017). HSPA5 regulates ferroptotic cell death in cancer cells. Cancer Res. 77, 2064-2077. doi: 10.1158/0008-5472.can-16-1979

Zille, M., Karuppagounder, S. S., Chen, Y., Gough, P. J., Bertin, J., Finger, J., et al. (2017). Neuronal death after hemorrhagic stroke in vitro and in vivo shares features of ferroptosis and necroptosis. Stroke 48, 1033-1043. doi: 10.1161/ strokeaha.116.015609

Conflict of Interest: The authors declare that the research was conducted in the absence of any commercial or financial relationships that could be construed as a potential conflict of interest.

Copyright (c) 2020 Liu, Guo, Yan, Huang, Ren, Luo and Yang. This is an openaccess article distributed under the terms of the Creative Commons Attribution License (CC BY). The use, distribution or reproduction in other forums is permitted, provided the original author(s) and the copyright owner(s) are credited and that the original publication in this journal is cited, in accordance with accepted academic practice. No use, distribution or reproduction is permitted which does not comply with these terms. 